\title{
Wind-Driven Oscillations in Meridional Overturning Circulations near the Equator. Part II: Idealized Simulations 0
}

\author{
Michael J. Bell, ${ }^{a}$ AdAM T. Blaker, ${ }^{\mathrm{b}}$ AND JoËL J.-M. HirsChi ${ }^{\mathrm{b}}$ \\ ${ }^{\text {a }}$ Met Office, Exeter, United Kingdom \\ ${ }^{\mathrm{b}}$ National Oceanography Centre, Southampton, United Kingdom
}

(Manuscript received 28 November 2019, in final form 21 December 2020)

\begin{abstract}
Large-amplitude $\left[ \pm 100 \mathrm{~Sv}\left(1 \mathrm{~Sv} \equiv 10^{6} \mathrm{~m}^{3} \mathrm{~s}^{-1}\right)\right]$, high-frequency oscillations in the Pacific Ocean's meridional overturning circulation within $10^{\circ}$ of the equator have been found in integrations of the NEMO ocean general circulation model. Part I of this paper showed that these oscillations are dominated by two bands of frequencies with periods close to 4 and 10 days and that they are driven by the winds within about $10^{\circ}$ of the equator. This part shows that the oscillations can be well simulated by small-amplitude, wind-driven motions on a horizontally uniform, stably stratified state of rest. Its main novelty is that, by focusing on the zonally integrated linearized equations, it presents solutions for the motions in a basin with sloping side boundaries. The solutions are found using vertical normal modes and equatorial meridional modes representing Yanai and inertia-gravity waves. Simulations of 16-day-long segments of the time series for the Pacific of each of the first three meridional and vertical modes (nine modes in all) capture between $85 \%$ and $95 \%$ of the variance of matching time series segments diagnosed from the NEMO integrations. The best agreement is obtained by driving the solutions with the full wind forcing and the full pressure forces on the bathymetry. Similar results are obtained for the corresponding modes in the Atlantic and Indian Oceans. Slower variations in the same meridional and vertical modes of the MOC are also shown to be well simulated by a quasi-stationary solution driven by zonal wind and pressure forces.
\end{abstract}

KEYWORDS: Inertia-gravity waves; Internal waves; Meridional overturning circulation; Oscillations

\section{Introduction}

Much research into the meridional overturning circulation (MOC) focuses on mid- to high latitudes in the North Atlantic Ocean and the Southern Ocean, because these are the sources of the headwaters of the North Atlantic Deep Water (NADW) and of the Antarctic Bottom Water (AABW) (e.g., Sloyan and Rintoul 2001; Marshall and Speer 2012; Cunningham et al. 2007; Smeed et al. 2018; Lozier et al. 2019; Johnson et al. 2019). However, the MOC in equatorial regions has also been recognized as playing an important role in the climate system (e.g., McPhaden and Zhang 2002; Zhang and McPhaden 2006; Vecchi and Soden 2007; Song et al. 2018). There are several motivations for studying MOCs near the equator.

First, the MOC in the Pacific Ocean equatorial region is thought to interact with phenomena such as the El NiñoSouthern Oscillation (ENSO) or the Pacific decadal oscillation (PDO) with impact on the uptake or outgassing of $\mathrm{CO}_{2}$ in the equatorial region (e.g., McPhaden and Zhang 2002; Gruber et al. 2009; Song et al. 2018). During La Niña conditions, the wind-driven equatorial upwelling in the eastern Pacific allows a persistent heat flux from the atmosphere to the ocean to be maintained there. Clement et al. (1996) and Cane et al. (1997) have suggested that this upwelling could retard the warming of

Supplemental information related to this paper is available at the Journals Online website: https://doi.org/10.1175/JPO-D-190297.s1.

Corresponding author: Michael J. Bell, mike.bell@metoffice. gov.uk the eastern Pacific obtained under climate change. Andrews and Webb (2018) explain how different patterns of warming along the equatorial Pacific are associated with different cloud feedbacks and different equilibrium climate sensitivities (the global SST warming for a given increase in greenhouse gas forcing).

Second, in ocean models the equatorial MOC stands out as having high variability on short (days to seasonal) time scales. The large seasonal variability can readily be explained by changes in the wind-driven Ekman overturning cells associated with the seasonal movement of the intertropical convergence zone (e.g., Lee and Marotzke 1998). In addition to this wellknown and understood variability in the MOC the equatorial region has also been shown to exhibit a very large (peak to peak of up to several hundred Sverdrups) MOC variability on time scales shorter than 10 days (e.g., Hirschi et al. 2013, 2020; Blaker et al. 2021, hereafter Part I).

Third, in ocean reanalyses unreliable meridional overturning circulations can occur in the vicinity of the equator, generated by the assimilation of oceanographic measurement data into ocean models (Park et al. 2018). Therefore, several reanalyses take special steps to attempt to control these artifacts (Bell et al. 2004; Balmaseda et al. 2013; Waters et al. 2014). Nonetheless the initialization of ENSO seasonal forecasts remains unsatisfactory, as it is difficult to decide how to retain the impact of ocean profile measurements into the forecasts (Mulholland et al. 2016). Any new insights into the dynamics that control the depth and strength of the equatorial MOCs would thus be extremely valuable.

This is the second part of a two-part paper seeking to interpret the large-amplitude oscillations in the MOC found near the equator in global NEMO simulations in the Atlantic, 

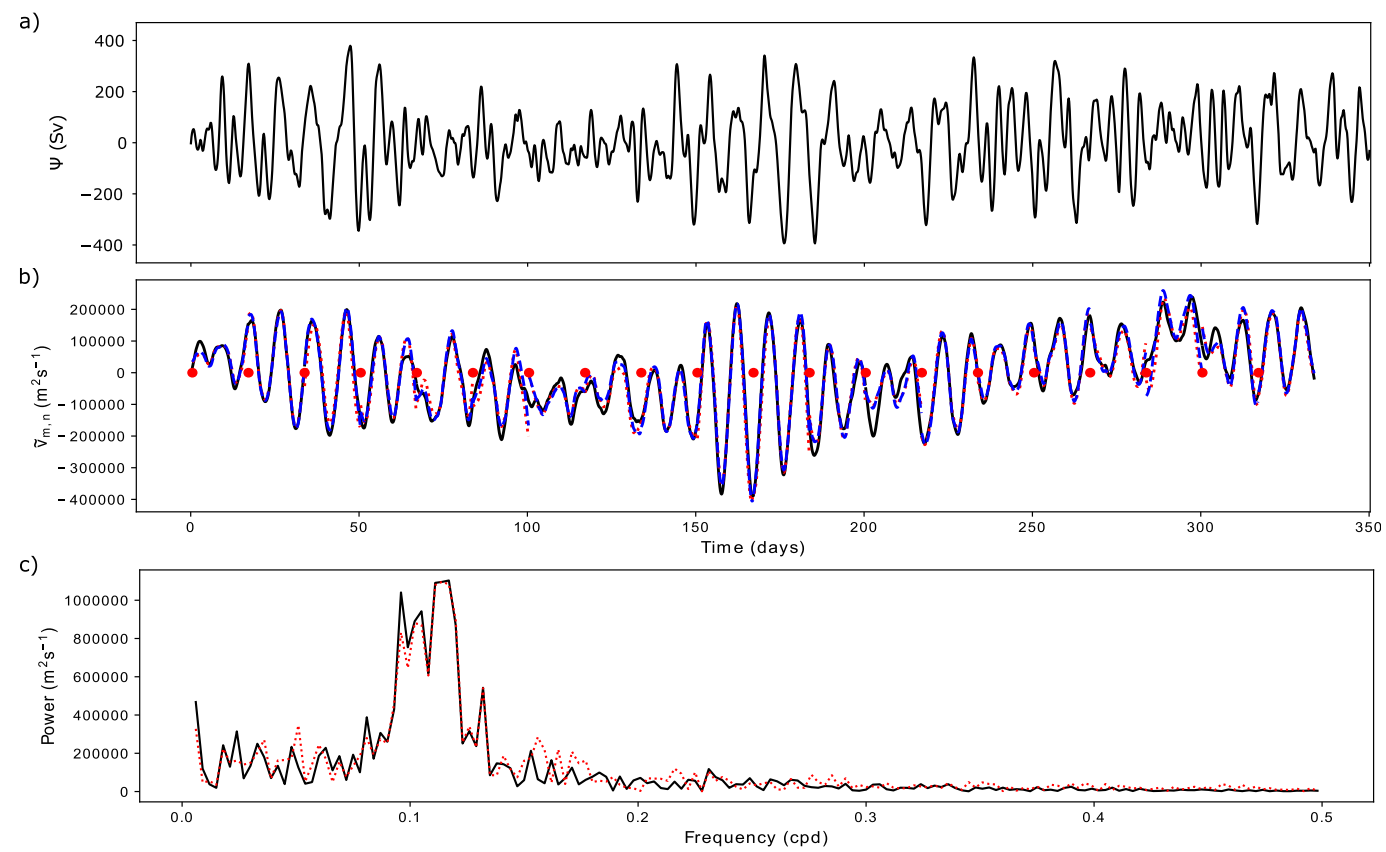

FIG. 1. (a) Time series of the Pacific MOC (Sv; $\left.1 \mathrm{~Sv} \equiv 10^{6} \mathrm{~m}^{3} \mathrm{~s}^{-1}\right)$ on the equator at $1583-\mathrm{m}$ depth from the NEMO integration. (b) Time series of the amplitudes $\left(\mathrm{m}^{2} \mathrm{~s}^{-1}\right)$ of meridional mode zero $(n=0)$, first baroclinic vertical mode $(m=1)$ for the Pacific Ocean in the NEMO integrations (black), the "natural" simulation $D_{N}$ (red dashed), and least squares fit simulation $D_{H}$ (blue). The simulations are restarted every 100 time steps, at the times marked by red circles. (c) Power spectra of the black (NEMO) and red (natural simulation) time series shown in (b).

Indian, and Pacific Ocean basins initially reported by Hirschi et al. (2013). Figure 1a illustrates these oscillations with a 1-yr time series for the Pacific MOC on the equator at $1583-\mathrm{m}$ depth calculated from 4-h time mean velocities. Part I shows that these oscillations are driven by the winds within $10^{\circ}$ of the equator and that their periods, typically between 4 and 10 days, correspond to those of the long zonal wavelength, low vertical wavenumber, baroclinic Yanai and inertia-gravity waves identified within TAO mooring data by Farrar and Durland (2012). Part I shows that the oscillations are not associated with boundary currents and that the amplitude of the MOC variability is roughly proportional to the width of the ocean basin (approximately $0.03 \mathrm{~Sv} \mathrm{~km}^{-1} ; 1 \mathrm{~Sv} \equiv 10^{6} \mathrm{~m}^{3} \mathrm{~s}^{-1}$ ). Although the oscillations in the MOC transport are large $( \pm 200 \mathrm{~Sv}$ in the Pacific Ocean), the meridional velocities associated with them, if they occur across the whole basin and down to depths of $1500 \mathrm{~m}$, could be little more than about $1 \mathrm{~cm} \mathrm{~s}^{-1}$.

In this part of the paper we aim to simulate these oscillations as "classical" small-amplitude, wind-driven motions on a horizontally uniform, stably stratified, state of rest, as described by Gill (1982). McCreary (1985) and Clarke (2008) provide useful reviews of this approach to equatorial waves that was made popular by the seminal paper of Lighthill (1969). The wind forcing is projected onto the vertical normal modes as in the modeling of atmospheric tides (Chapman and Lindzen 1970). The response to a sudden change in the wind forcing in an unbounded ocean is one topic of interest. Cane and Sarachik (1976) study this with particular attention to the asymptotic response at the head of the resulting train of planetary waves.
Another topic of interest is the response to an equatorial Kelvin wave impinging on an eastern boundary. Moore (1968), Anderson and Rowlands (1976) and Cane and Sarachik (1979) study this with particular attention to the resulting coastal propagation and westward propagation of planetary waves. In both cases the dispersion relations for meridional equatorial modes discovered by Blandford (1966) play important roles in the simplification and interpretation of the Laplace transforms used in the solutions.

The solutions in the above papers are restricted to oceans with flat bottoms, any side boundaries being strictly vertical. In this paper, motivated by the results presented in Part I, we focus solely on zonally integrated motions. As explained in section 2, this allows us to use vertical normal modes in ocean basins with sloping bathymetries, the only restriction on the bathymetry being that its maximum depth $H_{\max }$ at a given latitude is in fact independent of latitude. We also focus mainly on high-frequency motions while most of the literature cited above focuses on the slower planetary wave solutions. It turns out that the dispersion relations for the zonally integrated motions are somewhat simpler than the standard case and that the time dependence of the amplitudes of the modes is determined by an equation for a wind-driven harmonic oscillator. So we do not need to resort to Laplace transforms, Green's functions, or Fourier transforms in our solutions.

Figure $1 \mathrm{~b}$ shows that the solutions of the resulting equations reproduce very well the time series from the NEMO integration of the zonal integral of the meridional velocity in the first baroclinic mode and meridional mode zero. Using the 
linearized simulations derived in this paper it was shown in Part I that the first six meridional and vertical modes are able to account for a large fraction of the variability in the MOC within $10^{\circ}$ of the equator.

The paper is structured as follows. Section 2 describes the derivation of the linearized normal-mode solutions. Section 3 describes the methods used to integrate the linearized equations and to assess the quality of their solutions. It also describes the simulations that are presented. Section 4 describes the results. Section 5 summarizes the main conclusions and discusses some open issues.

\section{Derivation of the linearized normal-mode solutions}

\section{a. Overview of derivation}

We will consider motions driven by time-varying winds, with zonal and meridional components, in a basin straddling the equator as illustrated by the schematic in Fig. 2. As in the schematic, the bathymetry of the basin may be quite complex but its lateral boundaries are assumed to be closed (e.g., the Indonesian Throughflow is neglected). Following Gill (1982), we assume that the motions can be considered to be smallamplitude fluctuations about a horizontally uniform, stably stratified, state of rest. [Of course in "reality" the depth of the thermocline in the eastern equatorial Pacific is considerably shallower than that in the western equatorial Pacific, there are marked north-south variations in the depth of the thermocline near the equator, and there are zonal currents along the equator (and to its north and south) with strength approaching $1 \mathrm{~m} \mathrm{~s}^{-1}$. It is nonetheless assumed that, for low vertical wavenumber modes, these variations in the stratification and zonal currents can be neglected. The adequacy of this approach is discussed in sections $4 a$ and 5].

Our derivation of the linearized equations governing the motions is presented in section $2 \mathrm{~b}$. Its main novelty is that it starts by integrating the equations of motion zonally across the ocean basin. This allows the bathymetry to vary with latitude and longitude and the solutions to be written in terms of the zonally integrated transports. It also makes it clear that the solutions satisfy the condition of no normal flow at the boundaries of the basin. It simplifies the equations by eliminating zonal variations but implies that the pressure forces on the boundaries as well as the surface wind stresses drive the motions. This approach would be somewhat unsatisfactory if these pressure forces dominated the wind stresses at the frequencies of interest. We show that they do not in section 4c. It turns out that the solutions involve only second-order, not third-order, time derivatives [for reasons explained in the discussion following (34)]. The zonally integrated linearized equations are solved by projecting the wind stresses and pressure forcing onto the vertical and meridional normal modes. Sections $2 \mathrm{c}$ and $2 \mathrm{~d}$ derive the projections onto the vertical and meridional modes, respectively. The time evolution of the amplitude of the meridional velocities of the modes is found to be determined by an equation, (33), that describes a simple harmonic oscillator driven by wind and pressure forcing. Section $2 \mathrm{e}$ provides summaries of the derivation and the procedure for calculating the solutions.

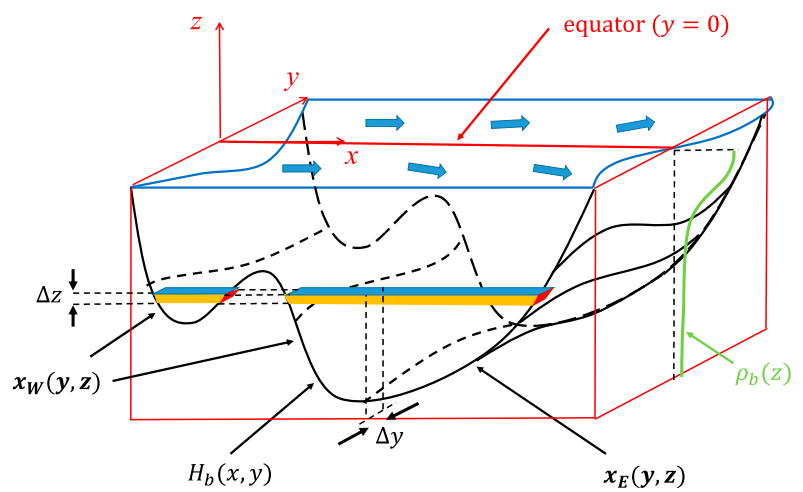

FIG. 2. Schematic depiction of the ocean basin. Axes and lines parallel to them, including the equator, are colored red. Surface fields, including thick arrows for the surface wind stresses, are colored blue. The bathymetry is colored black, and the basic state density profile is green. The depth of the bottom $H_{b}$ may depend on $x$ and $y$, and the surface wind stress vector $\left(X_{s}, Y_{s}\right)$ may depend on $x, y$, and $t$. The eastern and western sides of the basin $x_{E}$ and $x_{W}$, respectively, may depend on $y$ and $z$, and the basic state density $\rho_{b}$ may depend on $z$. A zonal integral over the volume of width $\Delta y$ and height $\Delta z$ is used in (8) to derive (4).

\section{b. Governing equations}

For simplicity Cartesian coordinates are used with $x, y$, and $z$ directed eastward, northward and upward, respectively. The depth $H_{b}$ of the basin is allowed to vary [i.e., $\left.H_{b}(x, y)\right]$ as are the "longitudes" of the eastern and western boundaries [i.e., $x_{E}(y, z)$, $\left.x_{W}(y, z)\right]$. Denoting the maximum depth of the basin at a given latitude by $H_{\max }(y)$, our solution technique requires that $H_{\max }$ be independent of $y$. This depth is denoted as $H$. Smallamplitude variations are denoted by primes, and quantities that are zonally integrated across the basin are denoted by angle brackets (e.g., $\left\langle u^{\prime}\right\rangle$ is the zonal integral of $u^{\prime}$ ). As illustrated in Fig. 2 the zonal integral for given $y$ and $z$ may have $N$ segments, $N$ eastern and $N$ western boundaries $(N=$ 2 below the midocean ridge in the figure). The zonal, meridional, and vertical velocities of the small-amplitude motions are denoted by $u^{\prime}, v^{\prime}$, and $w^{\prime}$, respectively and $p^{\prime}$ denotes pressure perturbations; $p_{E}^{\prime}$ and $p_{W}^{\prime}$ denote the pressure perturbations evaluated on the eastern and western boundaries. The zonal and meridional components of the downward flux of momentum input by the winds are denoted by $X^{\prime}(x, y, z, t)$ and $Y^{\prime}(x, y, z, t)$, and their surface values (the surface wind stress) are denoted by $X_{s}(x, y, t)$ and $Y_{s}(x, y, t)$. The potential density is denoted by $\rho, \rho_{0}$ is a constant density $\left(1026 \mathrm{~kg} \mathrm{~m}^{-3}\right)$, and $\rho_{b}(z)$ represents the stably stratified density profile of the basic state.

The hydrostatic, incompressible equations for large horizontal scales of motion, zonally integrated across the basin illustrated in Fig. 2 are then

$$
\begin{aligned}
& \frac{\partial\left\langle u^{\prime}\right\rangle}{\partial t}+r\left\langle u^{\prime}\right\rangle-f\left\langle v^{\prime}\right\rangle=\rho_{o}^{-1}\left(\left\langle\frac{\partial X^{\prime}}{\partial z}\right\rangle+p_{W}^{\prime}-p_{E}^{\prime}\right), \\
& \frac{\partial\left\langle v^{\prime}\right\rangle}{\partial t}+r\left\langle v^{\prime}\right\rangle+f\left\langle u^{\prime}\right\rangle=-\rho_{o}^{-1}\left(\frac{\partial\left\langle p^{\prime}\right\rangle}{\partial y}-\mathcal{A}\right)+\rho_{o}^{-1}\left\langle\frac{\partial Y^{\prime}}{\partial z}\right\rangle,
\end{aligned}
$$




$$
\begin{aligned}
\frac{\partial\left\langle p^{\prime}\right\rangle}{\partial z}-\mathcal{B} & =-\left\langle\rho^{\prime}\right\rangle g \\
\frac{\partial\left\langle v^{\prime}\right\rangle}{\partial y}+\frac{\partial\left\langle w^{\prime}\right\rangle}{\partial z} & =0, \quad \text { and } \\
\frac{\partial\left\langle\rho^{\prime}\right\rangle}{\partial t}+\left\langle w^{\prime}\right\rangle \frac{\partial \rho_{b}}{\partial z} & =0 .
\end{aligned}
$$

In the above $f=\beta y$ is the Coriolis parameter and $r$ is a weak Rayleigh damping coefficient that crudely represents the effects of unresolved turbulent motions; use of Rayleigh friction is consistent with application of boundary conditions of no normal flow. The terms

$$
\mathcal{A} \equiv p_{E}^{\prime} \frac{\partial x_{E}}{\partial y}-p_{W}^{\prime} \frac{\partial x_{W}}{\partial y} \quad \text { and } \quad \mathcal{B} \equiv p_{E}^{\prime} \frac{\partial x_{E}}{\partial z}-p_{W}^{\prime} \frac{\partial x_{W}}{\partial z}
$$

in (2) and (3) arise because, for example,

$$
\frac{\partial\left\langle p^{\prime}\right\rangle}{\partial y}=\left\langle\frac{\partial p^{\prime}}{\partial y}\right\rangle+\frac{\partial x_{E}}{\partial y} p_{E}^{\prime}-\frac{\partial x_{W}}{\partial y} p_{W}^{\prime} .
$$

In deriving (4) we have zonally integrated the incompressibility condition over the volume segments with width $\Delta y$ and height $\Delta z$ illustrated in Fig. 2 (the integral illustrated has two segments). Using the divergence theorem and applying the boundary conditions of no normal flow on the sloping side boundaries (the faces colored red where they are visible), one sees that the integral receives contributions only from the four other faces (the ones colored blue and yellow where they are visible). Taking limitingly small values of $\Delta y$ and $\Delta z$,

$$
\begin{aligned}
I & \equiv \int_{z}^{z+\Delta z} \int_{y}^{y+\Delta y} \int_{x_{W}}^{x_{E}}\left(\frac{\partial u^{\prime}}{\partial x}+\frac{\partial v^{\prime}}{\partial y}+\frac{\partial w^{\prime}}{\partial z}\right) d x d y d z \\
& =\Delta z \int_{x_{W}(y+\Delta y, z)}^{x_{E}(y+\Delta y, z)} v^{\prime}(y+\Delta y, z) d x-\Delta z \int_{x_{W}(y, z)}^{x_{E}(y, z)} v^{\prime}(y, z) d x+\Delta y \int_{x_{W}(y, z+\Delta z)}^{x_{E}(y, z+\Delta z)} w^{\prime}(y, z+\Delta z) d x-\Delta y \int_{x_{W}(y, z)}^{x_{E}(y, z)} w^{\prime}(y, z) d x
\end{aligned}
$$

Then using the definitions of the zonal integrals

$$
\begin{aligned}
I= & \Delta z\left[\left\langle v^{\prime}(y+\Delta y, z)\right\rangle-\left\langle v^{\prime}(y, z)\right\rangle\right] \\
& +\Delta y\left[\left\langle w^{\prime}(y, z+\Delta z)\right\rangle-\left\langle w^{\prime}(y, z)\right\rangle\right] \\
= & \Delta y \Delta z\left(\frac{\partial\left\langle v^{\prime}\right\rangle}{\partial y}+\frac{\partial\left\langle w^{\prime}\right\rangle}{\partial z}\right) .
\end{aligned}
$$

The zonal component of the momentum equation involves the pressures on the eastern and western boundaries. Fluctuations in these pressures near the equator are likely to result from Kelvin and Rossby waves driven by winds along the equator or winds near the boundaries. We will assume that the momentum fluxes input by the winds $\left(X^{\prime}, Y^{\prime}\right)$ and also the pressure fluctuations, $p_{E}^{\prime}$ and $p_{W}^{\prime}$, on the eastern and western boundaries are known. Separate calculations similar to those described in section 11.11 of Gill (1982) (see also Clarke 2008) would be needed to calculate $p_{E}^{\prime}$ and $p_{W}^{\prime}$ from the wind stresses.

In the following derivations we set the terms $\mathcal{A}$ and $\mathcal{B}$, which are defined by (6) and only appear in (2) and (3), to zero. We make this choice to avoid complicating the presentation of the algebra and because we are able to simulate the motions well by using calculations that omit these terms. Appendix A explains how the corresponding forcing terms could be calculated from the NEMO ocean model output and suggests why the terms do not contribute more substantially to the fluctuations.

We can use (3) in (5) to eliminate $\left\langle\rho^{\prime}\right\rangle$ :

$$
\frac{\partial^{2}\left\langle p^{\prime}\right\rangle}{\partial z \partial t}+\rho_{0} N^{2}\left\langle w^{\prime}\right\rangle=0, \text { where } N^{2}=-\frac{g}{\rho_{0}} \frac{\partial \rho_{b}}{\partial z} .
$$

This leaves four equations, (1), (2), (4), and (9) for four unknowns, $\left\langle u^{\prime}\right\rangle,\left\langle v^{\prime}\right\rangle,\left\langle w^{\prime}\right\rangle$, and $\left\langle p^{\prime}\right\rangle$. For brevity in (1) we will write

$$
\Delta p^{\prime} \equiv p_{W}^{\prime}-p_{E}^{\prime}
$$

\section{c. Projection onto vertical normal modes}

Section 6.11 of Gill (1982) describes the projection of solutions to the equations of motion for large horizontal scales onto the orthogonal vertical normal modes; the basic state and resulting equations are sufficiently simple that the solutions can be found by the method of separation of variables. Following Gill's notation, functions that depend only on $z$ are denoted by a circumflex [e.g., $\hat{h}(z)]$ and those that depend only on $y$ and $t$ by a tilde [e.g., $\tilde{w}(y, t)]$. The solutions of our equations can be written as linear sums of products of functions of $z$ and functions of $y$ and $t$ :

$$
\begin{aligned}
\left\langle w^{\prime}\right\rangle & =\sum_{m} \hat{h}_{m}(z) \tilde{w}_{m}(y, t) \quad \text { and } \\
{\left[\left\langle p^{\prime}\right\rangle, \rho_{0} g\left\langle u^{\prime}\right\rangle, \rho_{0} g\left\langle v^{\prime}\right\rangle\right] } & =\sum_{m} \hat{p}_{m}(z)\left[\tilde{h}_{m}, \tilde{u}_{m}, \tilde{v}_{m}\right] .
\end{aligned}
$$

Here the square bracket notation $\left[a_{1}, a_{2}, a_{3}\right]=\left[b_{1}, b_{2}, b_{3}\right]$ means $a_{1}=b_{1}, a_{2}=b_{2}$ and $a_{3}=b_{3}$. The functions $\tilde{w}_{m}, \tilde{h}_{m}, \tilde{u}_{m}$ and $\tilde{v}_{m}$, which depend only on $y$ and $t$, and $\hat{h}_{m}$ and $\hat{p}_{m}$, which depend only on $z$, are yet to be determined. For the projection of the wind and pressure forcing onto these normal modes we set

$$
\left[g \frac{\partial\left\langle X^{\prime}\right\rangle}{\partial z}, g \frac{\partial\left\langle Y^{\prime}\right\rangle}{\partial z}, \Delta p^{\prime}\right]=\sum_{m} \hat{p}_{m}(z)\left[\tilde{X}_{m}, \tilde{Y}_{m}, \Delta \tilde{h}_{m}\right],
$$

where again $\tilde{X}_{m}, \tilde{Y}_{m}$ and $\Delta \tilde{h}_{m}$ depend only on $y$ and $t$. We will assume a simple (but realistic) profile for the input of momentum by the wind stress into the mixed layer later in this subsection. One is free to choose the dimensions of either $\hat{h}_{m}$ or $\hat{p}_{m}$. We choose the dimensions of $\hat{h}_{m}$ to be the same as $z$ (i.e., meters). The dimensions of $\hat{p}_{m}$ are then the same as those of $\rho_{0} g$ and the dimensions of $\tilde{u}_{m}$ and $\tilde{v}_{m}$ are the same as those of $\left\langle u^{\prime}\right\rangle$ (i.e., $\mathrm{m}^{2} \mathrm{~s}^{-1}$ ). 
Substituting (11) and (12) in the four equations for the four dependent variables one obtains two separate sets of equations. Choosing

$$
\tilde{w}_{m}=\partial \tilde{h}_{m} / \partial t
$$

the vertical variations must satisfy

$$
\frac{d \hat{p}_{m}}{d z}=-\rho_{0} N^{2} \hat{h}_{m} \quad \text { and } \quad \hat{p}_{m}=\rho_{0} g H_{m} \frac{d \hat{h}_{m}}{d z}
$$

where $H_{m}$ is a constant of separation. To satisfy $\left\langle w^{\prime}\right\rangle=0$ at the deepest point of the basin $z=-H_{\max }$, as stated earlier we require $H_{\max }$ to be independent of $y$. Then, denoting $H_{\max }$ by $H$, we require $\hat{h}_{m}(-H)=0$. For the baroclinic modes the rigidlid approximation is very accurate, so we can also require $\hat{h}_{m}=0$ at the upper boundary:

$$
\hat{h}_{m}=0, \quad z=0,-H \text {. }
$$

The horizontal variations then satisfy the zonally integrated momentum equations

$$
\begin{aligned}
& \frac{\partial \tilde{u}_{m}}{\partial t}+r \tilde{u}_{m}-f \tilde{v}_{m}=\tilde{X}_{m}+g \Delta \tilde{h}_{m}, \\
& \frac{\partial \tilde{v}_{m}}{\partial t}+r \tilde{v}_{m}+f \tilde{u}_{m}=-g \frac{\partial \tilde{h}_{m}}{\partial y}+\tilde{Y}_{m},
\end{aligned}
$$

and

$$
\frac{\partial \tilde{h}_{m}}{\partial t}+H_{m} \frac{\partial \tilde{v}_{m}}{\partial y}=0
$$

Clearly (16) and (17) are the shallow water equations (for motions that are independent of $x$ ).

Denoting $g H_{m}$ by $c_{m}^{2},(14)$ and (15) gives a second-order ordinary differential equation (ODE) for $\hat{p}_{m}$

$$
\frac{d}{d z}\left(\frac{1}{N^{2}} \frac{d \hat{p}_{m}}{d z}\right)=-c_{m}^{-2} \hat{p}_{m}
$$

with boundary conditions

$$
\frac{d \hat{p}_{m}}{d z}=0, \quad z=0,-H
$$

These two equations are of Sturm-Liouville form with a unit weighting function (see e.g., Kreyszig 1979, his section 4.8). So the $\hat{p}_{m}$ are orthogonal eigenfunctions. We choose their amplitudes by writing them and the vertical coordinate $z$ in the nondimensional forms $\bar{p}_{m}$ and $\bar{z}$, respectively, where

$$
z=H \bar{z} \quad \text { and } \quad \hat{p}_{m}=\rho_{0} g \bar{p}_{m} .
$$

We normalize the nondimensional eigenfunctions $\bar{p}_{m}$ by setting

$$
\int_{-1}^{0} \bar{p}_{m} \bar{p}_{m^{\prime}} d \bar{z}=\delta_{m m^{\prime}}
$$

The wind stress forcing is taken to be imparted uniformly over the depth of the mixed layer $H_{M}$, so using (12) and (21)

$$
\begin{aligned}
\rho_{0}\left[\tilde{X}_{m}, \tilde{Y}_{m}\right] & =\frac{\left[\left\langle X_{s}\right\rangle,\left\langle Y_{s}\right\rangle\right]}{H_{M}} \int_{-\frac{H_{M}}{H}}^{0} \bar{p}_{m}(\bar{z}) d \bar{z}, \\
\rho_{0} g \Delta \tilde{h}_{m} & =\int_{-1}^{0} \bar{p}_{m}(\bar{z}) \Delta p^{\prime} d \bar{z},
\end{aligned}
$$

where (as stated earlier) $X_{s}$ and $Y_{s}$ are the zonal and meridional components of the surface wind stress and $\Delta p^{\prime}$, defined by (10), is the pressure difference between the eastern and western boundaries. In the simulations presented, $H_{M}$ is taken to be a constant (independent of space and time), but in principle this projection could use a mixed layer depth that depends on $x, y$, and $t$.

\section{d. Meridional modes}

Elimination of $\tilde{u}_{m}$ from (16) gives $\tilde{v}_{m}$ in terms of $\tilde{h}_{m}, \tilde{X}_{m}, \tilde{Y}_{m}$, and $\Delta \tilde{h}_{m}$ :

$$
\begin{aligned}
& {\left[\left(\frac{\partial}{\partial t}+r\right)^{2}+f^{2}\right] \tilde{v}_{m}} \\
& \quad=-f\left(\tilde{X}_{m}+g \Delta \tilde{h}_{m}\right)+\left(\frac{\partial}{\partial t}+r\right)\left(-g \frac{\partial \tilde{h}_{m}}{\partial y}+\tilde{Y}_{m}\right) .
\end{aligned}
$$

The main impact of the Rayleigh damping is to slowly reduce the amplitude of $\tilde{v}_{m}$ through the term proportional to $r$ on the lhs of (23). The coupling between modes that could be obtained by the term proportional to $r$ on the rhs of (23) is negligible unless two modes have almost the same frequency and hence interact resonantly. Neglecting that term and the term $r^{2} \tilde{v}_{m}$ on the lhs of (23) and using (17), one obtains

$$
\beta^{2} y^{2} \tilde{\boldsymbol{v}}_{m}-c_{m}^{2} \frac{\partial^{2} \tilde{\boldsymbol{v}}_{m}}{\partial y^{2}}=\frac{\partial \tilde{Y}_{m}}{\partial t}-f\left(\tilde{X}_{m}+g \Delta \tilde{h}_{m}\right)-\frac{\partial^{2} \tilde{\boldsymbol{v}}_{m}}{\partial t^{2}}-2 r \frac{\partial \tilde{\boldsymbol{v}}_{m}}{\partial t} .
$$

The $y$ dependence of the solutions can be separated from the time dependence by projecting the forcing onto the eigenfunctions of

$$
\frac{d^{2} \phi_{m, n}}{d y^{2}}-\left(\frac{\beta}{c_{m}}\right)^{2} y^{2} \phi_{m, n}=-\lambda_{m, n} \phi_{m, n},
$$

where $\lambda_{m, n}$ is a separation constant and the eigenvalue of the resulting second-order ODE.

Equation (25) is familiar from quantum mechanics as the equation governing the wavefunction for a harmonic oscillator. It has a set of complete orthogonal functions involving the Hermite polynomials and is reduced to normal form by introducing the nondimensional coordinates $\tilde{y}_{m}$ and functions $\tilde{\phi}_{m, n}$ defined by

$$
\tilde{y}_{m}=\left(\frac{2 \beta}{c_{m}}\right)^{1 / 2} y, \quad \tilde{\phi}_{m, n}\left(\tilde{y}_{m}\right)=\phi_{m, n}(y) .
$$

Equation (25) then transforms into the nondimensional form

$$
\frac{d^{2} \tilde{\phi}_{m, n}}{d \tilde{y}_{m}^{2}}-\frac{\tilde{y}_{m}^{2}}{4} \tilde{\phi}_{m, n}=-\frac{c_{m} \lambda_{m, n}}{2 \beta} \tilde{\phi}_{m, n} .
$$


The weighting function for the orthogonal functions is again unity, and the solutions are given by

$$
\tilde{\phi}_{m, n}\left(\tilde{y}_{m}\right)=A_{n} \exp \left(-\tilde{y}_{m}^{2} / 4\right) \mathrm{He}_{n}\left(\tilde{y}_{m}\right) \quad \text { and } \quad \frac{c_{m} \lambda_{m, n}}{2 \beta}=n+1 / 2 \text {. }
$$

Here $\operatorname{He}_{n}\left(\tilde{y}_{m}\right)$ are the probabilists' Hermite polynomials (section 4.9 of Kreyszig 1979):

$$
\begin{aligned}
& \mathrm{He}_{0}=1, \quad \mathrm{He}_{1}=\tilde{y}_{m}, \quad \mathrm{He}_{2}=\tilde{y}_{m}^{2}-1, \quad \text { and } \\
& \mathrm{He}_{3}=\tilde{y}_{m}^{3}-3 \tilde{y}_{m},
\end{aligned}
$$

and $A_{n}$ is a constant (independent of $\tilde{y}_{m}$ ) chosen so that

$$
\int_{-\infty}^{-\infty} \tilde{\phi}_{m, n}^{2}\left(\tilde{y}_{m}\right) d \tilde{y}_{m}=1 .
$$

The solutions of (24) can be written in the form

$$
\begin{aligned}
& {\left[\tilde{v}_{m}, f \tilde{X}_{m}, f \Delta \tilde{h}_{m}, \tilde{Y}_{m}\right]} \\
& \quad=\sum_{m=0}^{\infty}\left[\tilde{v}_{m, n}(t), \tilde{X}_{m, n}^{f}(t), \Delta \tilde{h}_{m, n}^{f}(t), \tilde{Y}_{m, n}(t)\right] \tilde{\phi}_{m, n}\left(\tilde{y}_{m}\right) .
\end{aligned}
$$

The $\tilde{X}_{m, n}^{f}(t), \Delta \tilde{h}_{m, n}^{f}(t)$, and $\tilde{Y}_{m, n}(t)$ can be calculated from the winds and boundary pressures by integrating meridionally over the basin and using the orthogonality and completeness of the solutions of (27) and (30):

$$
\begin{aligned}
& {\left[\tilde{X}_{m, n}^{f}(t), \Delta \tilde{h}_{m, n}^{f}(t), \tilde{Y}_{m, n}(t)\right]} \\
& \quad=\int_{-\infty}^{\infty}\left[f \tilde{X}_{m}(y, t), f \Delta \tilde{h}_{m}, \tilde{Y}_{m}(y, t)\right] \mathrm{He}_{m, n}\left(\tilde{y}_{m}\right) \exp \left(-\tilde{y}_{m}^{2} / 4\right) d \tilde{y}_{m} .
\end{aligned}
$$

This enables (24) to be reduced to a second-order ODE for $\tilde{v}_{m, n}(t)$ driven by the surface winds and pressures on the boundaries:

$$
\frac{d^{2} \tilde{\boldsymbol{v}}_{m, n}}{d t^{2}}+2 r \frac{d \tilde{v}_{m, n}}{d t}+c_{m}^{2} \lambda_{m, n} \tilde{\boldsymbol{v}}_{m, n}=\frac{d \tilde{Y}_{m, n}}{d t}-\left(\tilde{X}_{m, n}^{f}+g \Delta \tilde{h}_{m, n}^{f}\right) .
$$

Using (28), one sees that the natural, unforced and undamped, frequency $\omega_{m, n}$ of $\tilde{v}_{m, n}$ is given by

$$
\omega_{m, n}^{2}=c_{m}^{2} \lambda_{m, n}=\beta c_{m}(2 n+1)
$$

This result agrees with (11.6.7) of Gill (1982) for zonal wavenumber $k=0$. In many contexts $c_{m}=(g H)^{1 / 2}$ is a phase speed, and we will refer to it as such despite the absence of zonal phase propagation in our zonally integrated equations. The meridional mode with $n=0$ is a Yanai (mixed planetary-gravity) wave while the modes with $n>0$ are inertia-gravity waves. The dispersion relation (34) is only quadratic in $\omega_{m, n}$ whereas for equatorial waves with zonal wavenumber $k \neq 0$ it is cubic in $\omega_{m, n}$. This can be traced back to the absence of a horizontal pressure gradient term $-g\left(\partial \tilde{h}_{m} / \partial x\right)$ in the zonal component of (16). That term leads to one in (23) that is proportional to $f g\left(\partial \tilde{h}_{m} / \partial x\right)$. This forces differentiation of (23) w.r.t. time to eliminate $\partial h / \partial t$ using the continuity equation [(17)], which is not necessary for the zonally integrated case. The simpler dispersion relation can alternatively be interpreted by noting that the very long Kelvin and Rossby waves have zero frequency (i.e., $\omega=0$ when $k=0$ ).

The solutions for $\tilde{u}_{m, n}$ can be found from those for $\tilde{v}_{m, n}$ by using the zonal component of the momentum equations [the first of (16)]

$$
\frac{\partial \tilde{u}_{m}}{\partial t}+r \tilde{u}_{m}=\exp (-r t) \frac{\partial}{\partial t}\left[\exp (r t) \tilde{u}_{m}\right]=f \tilde{v}_{m}+\tilde{X}_{m}+g \Delta \tilde{h}_{m} .
$$

The projection of $f \tilde{v}=\beta y \tilde{v}$ onto the modes of $\tilde{u}$ can be calculated using (26) and the following identity satisfied by the Hermite polynomials

$$
\tilde{y} \mathrm{He}_{n}(\tilde{y})=\mathrm{He}_{n+1}(\tilde{y})+n \mathrm{He}_{n-1}(\tilde{y}) .
$$

\section{e. Summary of solution procedure}

In summary, (1)-(5) are solved by projecting the wind stress and pressure forcing onto the vertical and meridional normal modes. The vertical modes (numbered by $m$ ) are the solutions of (18) and (19) and the meridional modes (numbered $n$ ) are the trapped solutions of (27) given by (28) in which $\tilde{y}_{m}$ is a nondimensional form of $y$ defined by (26). The projection of the forcing onto the vertical modes is defined by (12) and calculated using (22). Its projection onto the meridional modes is defined by (31) and calculated using (32). The amplitudes $\tilde{v}_{m, n}$ of the zonally integrated velocities in mode $(m, n)$ are also defined by (31). They depend only on time, $t$, and evolve according to the forced harmonic oscillator equation [(33)] whose natural frequency $\omega_{m, n}$ is given by (34). The MOC is reconstructed from the $\tilde{\boldsymbol{v}}_{m, n}(t)$ and the normal modes, $\hat{p}_{m}(z)$ and $\tilde{\phi}_{m, n}(y)$, using (11) and (31).

\section{Description of methods and simulations}

\section{a. Description of NEMO integration}

The NEMO integration used is described in Blaker et al. (2012) and Part I. In brief the model uses an ORCA025 horizontal grid with isotropic grid spacing of about $1 / 4^{\circ}$ within $20^{\circ}$ of the equator and 75 vertical levels. It is forced by 6-hourly mean wind stresses and daily mean radiation and precipitation fields from the CORE2 interannually varying forcing (IAF) dataset (Large and Yeager 2009). For the period from 1 April 2006 to 31 March 2007 studied in this paper, the model outputs are stored as 4-hourly means.

\section{b. Calculation of basin geometry and forcing}

The western and eastern boundaries of each ocean basin are determined by following the land-sea boundary in the ocean model's land-sea mask at a chosen level northward or southward using the Pavlidis algorithm (chapter 7, section 5, of Pavlidis 1982). For all boundaries other than that of the western Pacific the level chosen is the surface. For that boundary the level chosen (at all latitudes) is at 1945-m depth. This is just 
below the sill of the Indonesian throughflow. There is hence a small normal flow through this boundary at shallower depths.

The zonal pressure gradient is recalculated at all the sea points for each model depth from the 4-hourly mean fields and integrated zonally to give the pressure force on the bathymetry for each model depth. Two calculations of the pressure forces on the bathymetry are used. First the full pressure force taking into account the variations in the sea surface height as well as variations in pressure with depth due to variations in density. Second, the pressure force excluding contributions from variations in sea surface height.

The 4-hourly NEMO meridional velocities are integrated zonally at each model depth to diagnose $\left\langle\boldsymbol{v}^{\prime}\right\rangle$ as a function of $y$ and $z$ and the 4-hourly wind stresses output by the model are similarly zonally integrated. The surface wind stress is assumed to decay linearly with depth over a mixed layer depth, $H_{m}=$ $50 \mathrm{~m}$ [see (22)]. This is shallower than the first zero depth of the vertical modes considered in this paper and the simulations for low vertical-mode numbers (e.g., $m=0$ and $m=1$ ) are relatively insensitive to variations in $H_{m}$ between $H_{m}=25 \mathrm{~m}$ and $H_{m}=75 \mathrm{~m}$. The simulations for higher vertical-mode numbers (e.g., $m=4$ and $m=5$ ) are somewhat sensitive to such variations in $H_{m}$.

\section{c. Time integration of $O D E S$}

Equation (33), determining the evolution of $\tilde{\boldsymbol{v}}_{m, n}(t)$, is a simple forced oscillator problem with natural frequency $\omega_{m, n}$ and damping coefficient $2 r$. The natural frequency of each mode is much larger than $r$ so the problem is that of a weakly damped oscillator that can respond vigorously when $\tilde{v}_{m, n}$ comes into the right phase with the forcing for a finite period of time.

As mentioned at the start of section $2 \mathrm{a}$ the stratification varies somewhat with latitude, longitude and time so one cannot precalculate the frequency of each mode exactly and should not expect the solutions to maintain the correct phase and amplitude for long periods. So solutions are generated for "short" integration periods and compared with the solutions diagnosed from the NEMO integrations. The solutions presented in this paper have been obtained using an integration period of 400 h (i.e., using $K=100$ data points).

The ODE (33) is solved by writing it as two coupled firstorder ODEs:

$$
\begin{aligned}
& \frac{d \tilde{v}_{m, n}}{d t}=\tilde{q}_{m, n}+\tilde{Y}_{m, n} \text { and } \\
& \frac{d \tilde{q}_{m, n}}{d t}=-2 r\left(\tilde{q}_{m, n}+\tilde{Y}_{m, n}\right)-c_{m}^{2} \lambda_{m, n} \tilde{v}_{m, n}-\left(\tilde{X}_{m, n}^{f}+\Delta \tilde{h}_{m, n}^{f}\right) .
\end{aligned}
$$

For each "short" integration period of $K$ points, spline fits are calculated for each of $\tilde{v}_{m, n}, \tilde{X}_{m, n}^{f}, \tilde{Y}_{m, n}$, and $\Delta \tilde{h}_{m, n}^{f}$ for a time series that extends three data points either side of the integration period. This allows initial values of $\tilde{q}_{m, n}$ and $\tilde{v}_{m, n}$ to be calculated and functions for calculating smooth values of $\tilde{X}_{m, n}$, $\tilde{Y}_{m, n}$, and $\Delta \tilde{h}_{m, n}^{f}$ during the integration period to be supplied to the function that integrates (37) over a chosen time period.

Appendix B describes a least squares method for choosing the initial conditions for $\tilde{q}_{m, n}$ and $\tilde{v}_{m, n}$ that minimizes the sum of the squares of the differences between the idealized simulation and the NEMO solution. This method is used by all the integrations presented in this paper.

\section{d. A least squares method to optimize the natural frequency, damping, and forcing}

The quality of the simulation of the oscillating time series can be expected to be sensitive to the specification of the natural frequency $\left[c_{m}^{2} \lambda_{m, n}\right.$ in (33)] and any constant error in the forcing. It will also depend somewhat on the amplitude of the forcing and the Rayleigh damping coefficient. To explore these issues it is useful to consider, instead of (33), the more general equation

$$
\begin{aligned}
\left(\frac{d^{2} \tilde{v}}{d t^{2}}\right)_{k}= & -2 r\left(\frac{d \tilde{v}}{d t}\right)_{k} \alpha_{0}-\omega^{2} \tilde{v}_{k} \alpha_{1}+\left(\frac{d \tilde{Y}}{d t}\right)_{k} \alpha_{2} \\
& -\tilde{X}_{k}^{f} \alpha_{3}-\Delta \tilde{h}_{k}^{f} \alpha_{4}+\alpha_{5}+\epsilon_{k} .
\end{aligned}
$$

Here the subscripts $m, n$ have been suppressed, the new subscript $k$ will be taken to indicate the time step, $\alpha_{0}-\alpha_{5}$ are constant scalar values, and $\epsilon_{k}$ is an error that depends on the time step. Equations (33) and (38) are identical if $\alpha_{i}=1$ for $i \leq$ $4, \alpha_{5}=0$, and $\epsilon_{k}=0$. Spline fits to the model solutions allow the first- and second-order time derivatives of the "true" model solution and the forcing to be calculated, so all the terms with a $k$ subscript other than $\epsilon_{k}$ can be taken to be known. Each of the constants $\alpha_{0}-\alpha_{5}$ can either be specified explicitly or taken to be unknown.

In a time segment of $K$ points there are $K$ instances of (38). If all six values $\alpha_{0}-\alpha_{5}$ are taken to be unknown, they can be estimated by minimizing the penalty function

$$
J_{1}=\sum_{k=1}^{K}\left[\left(\frac{d^{2} \tilde{\boldsymbol{v}}}{d t^{2}}\right)_{k}-\sum_{i=0}^{5} A_{k, i} \alpha_{i}\right]^{2}
$$

where

$$
\begin{aligned}
& A_{k, 0}=2 r\left(\frac{d \tilde{v}}{d t}\right)_{k}, \quad A_{k, 1}=\omega_{m, n}^{2} \tilde{v}_{k}, \quad A_{k, 2}=\left(\frac{d \tilde{Y}}{d t}\right)_{k}, \\
& A_{k, 3}=-\tilde{X}_{k}^{f}, \quad A_{k, 4}=-\Delta \tilde{h}_{k}^{f}, \quad \text { and } \quad A_{k, 5}=1 \text {. }
\end{aligned}
$$

Equation (38) is then integrated forward using these constant values from the initial conditions over the period of $K$ points as described in the previous subsection.

A very similar least squares fit approach is used to calculate $d \tilde{u}_{m, n} / d t$ from (35) and (36) given inputs of $\tilde{u}_{m, n}, \tilde{v}_{m, n-1}, \tilde{v}_{m, n+1}$ $\tilde{X}_{m, n}$, and $\Delta \tilde{h}_{m, n}$. The least squares fit can provide estimates of $r$ and the "best" coefficients multiplying $\tilde{\boldsymbol{v}}_{m, n-1}, \tilde{\boldsymbol{v}}_{m, n+1}$ and $\tilde{X}_{m, n}+\Delta \tilde{h}_{m, n}$, which we denote by $\gamma_{1}, \gamma_{2}$, and $\gamma_{3}$, respectively. Time series for $\tilde{u}_{m, n}$ are then obtained from time series for $\tilde{\boldsymbol{v}}_{m, n-1}, \tilde{\boldsymbol{v}}_{m, n+1}$, and $\tilde{X}_{m, n}+\Delta \tilde{h}_{m, n}$ and the fitted value of $r$ by integrating (35), choosing initial conditions that give the best fit.

\section{e. Calculation of slowly varying solutions}

A very simple solution of (33) can be obtained when the forcing is stationary: 
TABLE 1. Descriptions of the four types and three kinds of simulations performed and the statistical quantities calculated.

\begin{tabular}{cl}
\hline \hline & \\
\hline Type & No surface wind or pressure forcing \\
$A$ & Surface wind forcing only \\
$B$ & Surface wind and baroclinic pressure forcing \\
$C$ & Surface wind and full pressure forcing \\
$D$ & "Natural" solutions; no parameter adjustments \\
Kind & "Half fit" solutions; adjustment of natural frequency and constant offset \\
$N$ & "Full fit" solutions; adjustment of amplitude of forcings, natural frequency, and constant offset \\
$H$ & \\
$F$ & The mean variance of the time series segments \\
Statistic & The mean value of the fraction of the variance of the segments not accounted for by the simulated time series \\
$V_{T}$ &
\end{tabular}

$$
\tilde{v}_{m, n}=-\frac{\left(\tilde{X}_{m, n}^{f}+\Delta \tilde{h}_{m, n}^{f}\right)}{c_{m}^{2} \lambda_{m, n}} .
$$

We investigate to what extent slower variations in $\tilde{v}_{m, n}$ than those of the natural frequency are captured by (41). To do this we define the "slow" variations of a time series by its running mean over the period of the resonant frequency $\omega_{m, n}$. We compare the slow variations in time series of $\tilde{v}_{m, n}$ computed from the NEMO velocities with the slow variations in $\tilde{v}_{m, n}$ computed using (41).

The solution for $\tilde{\boldsymbol{v}}_{m, n}$ in (41) is independent of time when the forcing is stationary even when $r=0$. This case is of some interest because $r$ is likely to be weak. The zonal flow given by (35) has a steady solution when $r$ is nonzero, but when $r=0$ the zonal flow grows at a constant rate.

\section{f. Description of simulations performed}

Table 1 summarizes the four types of simulations of $\tilde{\boldsymbol{v}}_{m, n}$, denoted by the letters $A-D$, that will be presented. Simulations of type $A$ have no wind or pressure forcing. Type $B$ simulations are forced only by surface wind stresses. Type $C$ and type $D$ simulations are forced by wind stresses and pressures on the boundaries. Type $C$ simulations use the baroclinic pressure (not including the surface pressure), and type $D$ simulations use the full pressures. None of the simulations uses any Rayleigh damping, $r=0.0$, because the simulations are relatively short and the influence of Rayleigh damping is relatively weak in consequence.

Table 1 also summarizes the three kinds of simulations, denoted by subscripts $N, H$, and $F$, that may be presented for any type of simulation. Subscript $N$ (for natural) indicates that the simulations are solutions of (33) with the natural frequency, $\omega$ determined from (34). There is no "adjustment" of the forcing or natural frequency in these simulations. Subscript $H$ (for half fit) indicates that the simulations are solutions of (38) with the amplitudes of the forcing parameters $\left(\alpha_{2}, \alpha_{3}\right.$, and $\left.\alpha_{4}\right)$ set to 1 and only the parameters $\alpha_{1}$ and $\alpha_{5}$ (relating to the frequency and a constant offset, respectively) adjusted to find the best fit. Subscript $F$ (for full fit) indicates that the simulations are solutions of (38) with the five parameters $\left(\alpha_{1}-\alpha_{5}\right)$ adjusted to find the best fit. In all these simulations there is no Rayleigh damping $\left(\alpha_{0}=0\right)$.
The simulations of $\tilde{u}_{m, n}$ are of type $D$; that is, they are forced by surface winds and full pressures. Two kinds of simulation are presented. Both use the value of $r$ obtained using the least squares fit described in section $3 \mathrm{~d}$ above. Subscript $F$ indicates simulations in which the least squares fit coefficients $\gamma_{1}-\gamma_{3}$ are used to multiply the "forcing" terms in the time integration of (35). Simulations in which these three amplitudes are set to 1 are denoted by subscript $N$. For simplicity, both simulations use $\tilde{v}_{m, n}$ calculated directly from the NEMO integration.

\section{g. Description of statistics presented}

The statistics for $\tilde{\boldsymbol{v}}_{m, n}$ that are presented are based on the segments described in section 3c. The variance of the NEMO time series of $\tilde{v}_{m, n}$ is computed separately for each segment and its mean value, $V_{T}$ ( $T$ standing for time series), is taken to represent that of the whole time series. The variance of the difference between a simulation and the NEMO time series is also calculated separately for each segment. Its mean value $R_{T}$, divided by $V_{T}$, is denoted by $S_{T} ; S_{T}$ is the mean value of the fraction of the variance of the NEMO time series that is not accounted for by the simulated time series. The fraction of the variance that is accounted for by a time series is the square of the correlation coefficient $r_{C}$, so $r_{C}^{2}=1-S$ and $S_{T}=0.1$ corresponds to a value for $r_{C}=0.95$. For the reader's convenience, Table 1 provides a summary description of $V_{T}$ and $S_{T}$. Similar statistics have also been calculated for $d^{2} \tilde{\boldsymbol{v}}_{m, n} / d t^{2}$ but for reasons explained in section $4 \mathrm{f}$ the presentation here focuses on the fit of $\tilde{\boldsymbol{v}}_{m, n}$.

For the $\tilde{u}_{m, n}$ solutions, $V_{A}$ ( $A$ standing for acceleration) denotes the mean value of $(d \tilde{u} / d t)^{2}$ for the whole time series, $R_{A}$ denotes the mean of the square of the residual in $d \tilde{u} / d t$ after the least squares fit and $S_{A}=R_{A} / V_{A}$.

\section{Results}

\section{a. Sample time series}

Figure $1 \mathrm{~b}$ presents time series of $\tilde{\boldsymbol{v}}_{1,0}$, the amplitude of the first baroclinic vertical mode and meridional mode zero, $(m, n)=(1,0)$, in the Pacific for the full year starting on 1 April 2006. The black line is calculated directly from the NEMO meridional velocities. The red dashed lines are the natural solutions with full pressure forcing (simulation $D_{N}$ ) and the 

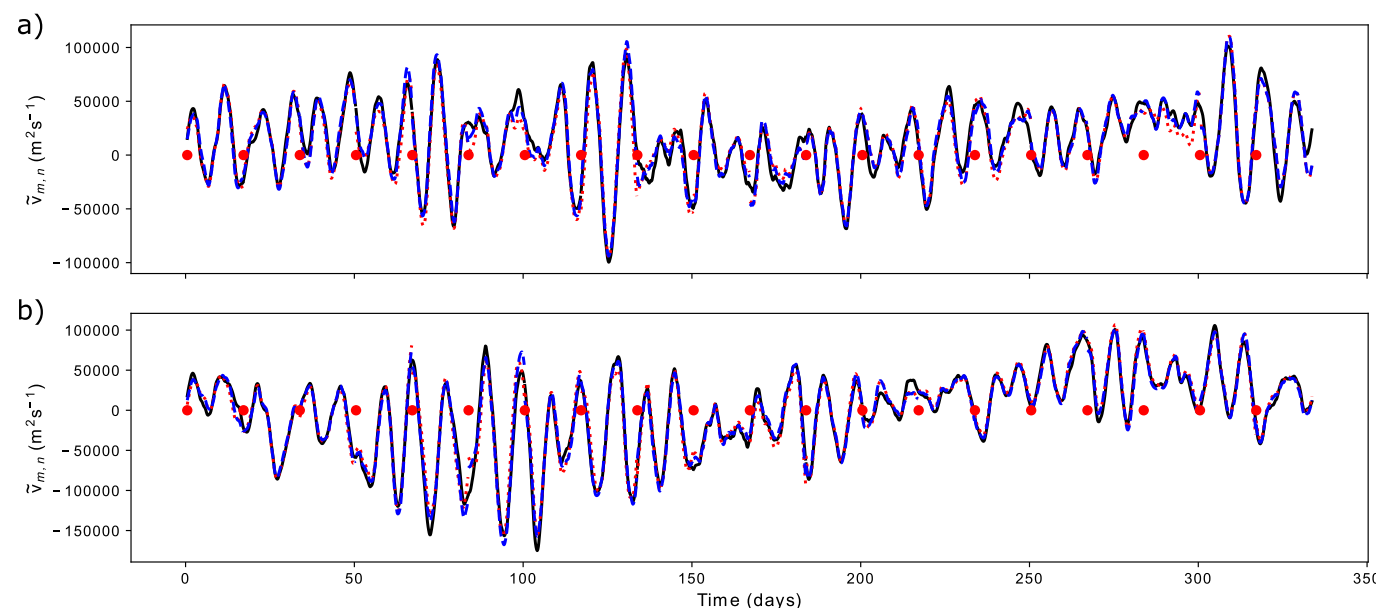

FIG. 3. The amplitudes of meridional mode zero $(n=0)$, first baroclinic vertical mode $(m=1)$ as in Fig. $1 \mathrm{~b}$, but for the (a) Atlantic and (b) Indian Oceans.

blue lines are the least squares fit solutions with full pressure forcing and the amplitude of the forcing held fixed (simulation $D_{H}$ ). The calculation of $c_{m}$ in the natural solutions, $D_{N}$, is detailed in the next subsection. The red circles indicate the start and end of the time segments; there are 20 time segments each containing 100 four-hour periods. The simulations generally follow the black curve very well, though there are some discrepancies visible around 100, 205, and 300 days. Simulations using integration periods that are twice as long (i.e., $800 \mathrm{~h}$ ) give time series that look qualitatively similar. Figure 1c presents the power spectra for the black and red lines presented in Fig. 1b. There is a high level of agreement particularly in the strongest peaks.

The time series for the Atlantic and Indian Oceans in Fig. 3 corresponding to those in Fig. 1b have similar characteristics. The idealized simulations again follow the NEMO simulations very well. The oscillations have frequencies and irregular variations in amplitude similar to those in the Pacific but the amplitude of the oscillations is larger in the Pacific than the other basins. Similarly high levels of agreement to those in Fig. $1 c$ are obtained for the power spectra of mode $(1,0)$ in the Atlantic and Indian Oceans (not shown).

Figure $4 \mathrm{a}$ is the same as Fig. 1b except that it presents results for mode $(m, n)=(2,2)$. These simulations are shown later to be typical of the poorest simulations for modes with $m \leq 3$ and $n \leq 2$. The frequency of oscillations in Fig. 4a is much higher than that in Fig. 1b, but the quality of the time series simulation still appears to be rather good. The agreement between the power series is again very good in the strongest peaks (not shown). Figure $4 \mathrm{~b}$ is the same as the top panel except that it presents mode $(m, n)=(5,3)$. As discussed later, this mode is one of the most poorly simulated ones. Simulation $D_{N}$ (red line) reproduces the modes quite well at times (e.g., from days 230 to 290 ) but poorly at others (e.g., from days 110 to 130 ).

The next three subsections investigate the dependence of the simulations and their quality in more detail. First, we quantify the dependence of the phase speeds, $c_{m}$, and hence the frequencies, $\omega_{m, n}$, on the background stratification. Second, we

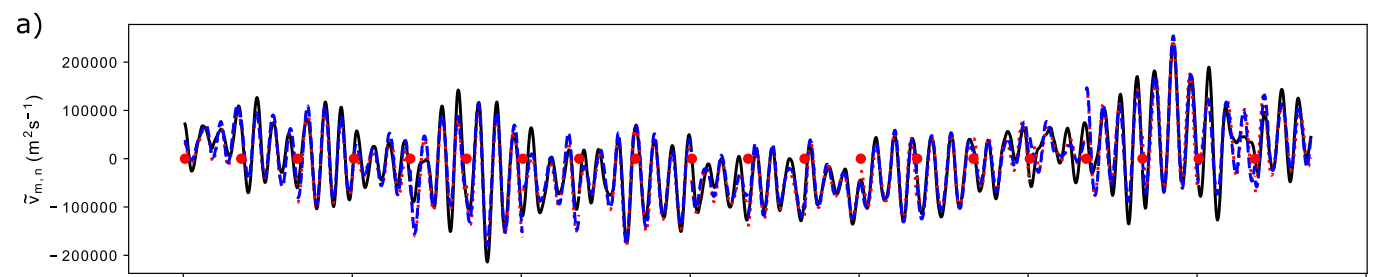

b)

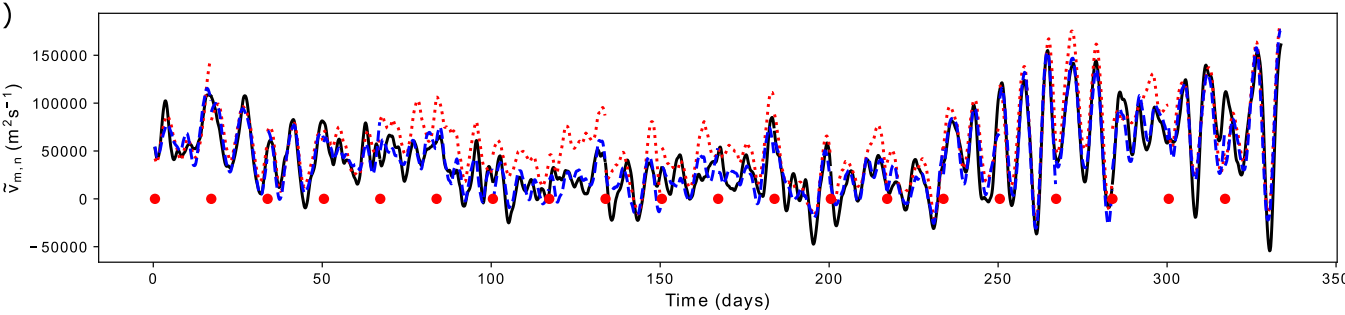

FIG. 4. As in Fig. 1b, but $(m, n)$ are equal to (a) $(2,2)$ and (b) $(5,3)$. 

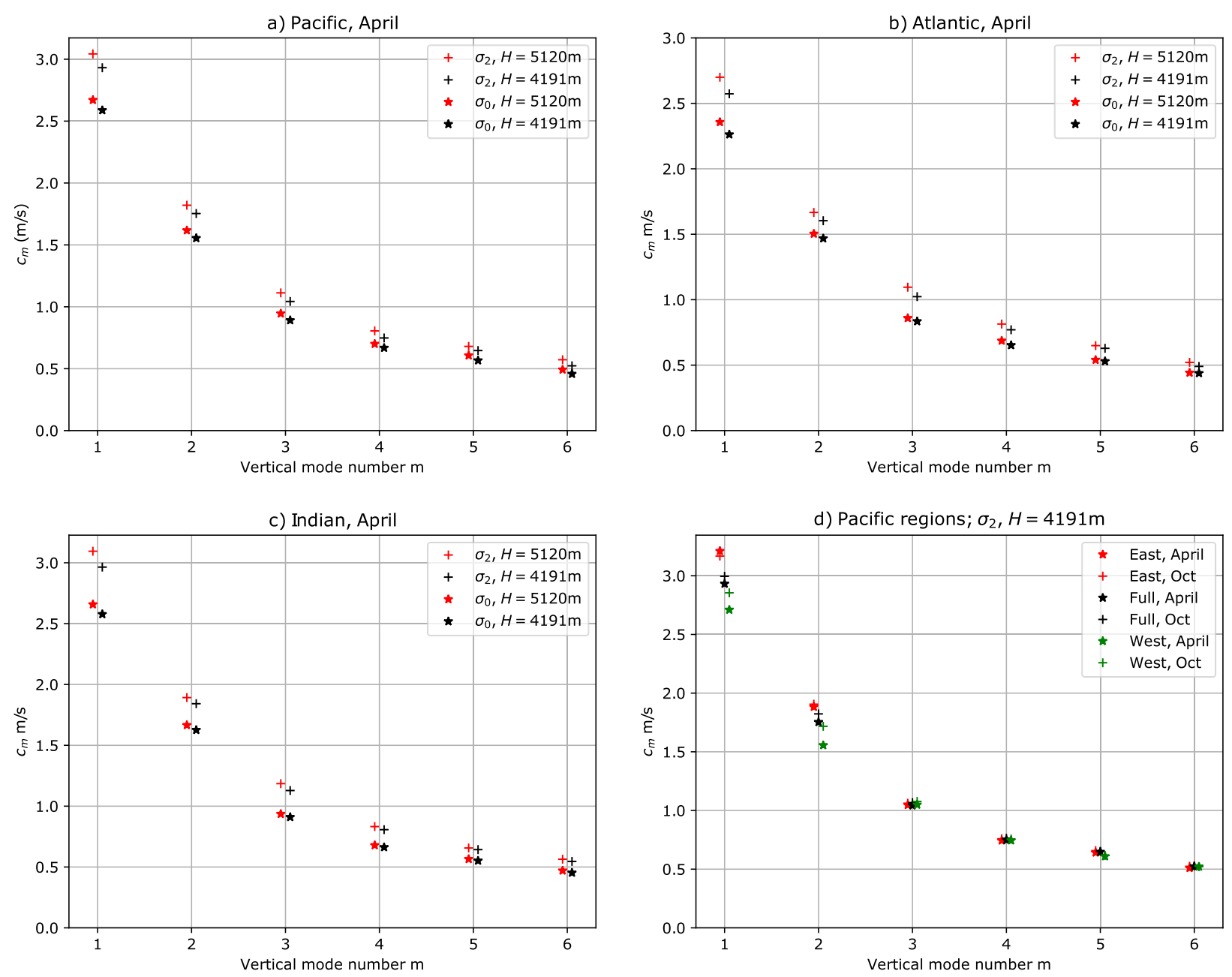

FIG. 5. Phase speeds of the first six baroclinic vertical modes $c_{m}$, calculated using April monthly mean data for the full (a) Pacific, (b) Atlantic, and (c) Indian Ocean basins. Values are plotted for two choices of $H$ and for potential density referred to the surface $\left(\sigma_{0}\right)$ and to $2000 \mathrm{~m}\left(\sigma_{2}\right)$. Also shown is (d) phase speeds for the Pacific calculated using $H=4191 \mathrm{~m}$ and $\sigma_{2}$ monthly mean potential densities for April or October and for the whole Pacific or its eastern or western halves. In all panels, values in different colors have been slightly offset along the $x$ axis to aid legibility.

show how the solutions depend on the kind and type of simulation. Third, we summarize how the quality of the simulations depends on the vertical- and meridional-mode numbers. We then illustrate how the modes contribute to the meridional overturning circulation. The last two subsections discuss the slow mode solutions for $\tilde{\boldsymbol{v}}_{m, n}$ and solutions for the zonal velocities.

\section{b. Calculation of vertical modes and their eigenvalues}

Figure 5 displays evaluations of the phase speeds $c_{m}$ of the first six baroclinic vertical modes computed using monthly mean zonally averaged data from the NEMO model integration giving equal weight to all data within $10^{\circ}$ of the equator. Values plotted in different colors have been slightly offset along the $x$ axis to aid legibility. Figure 5a displays values for the full width of the Pacific in April calculated using two alternative reference depths for the potential densities and two alternative "maximum" depths, $H$.
The phase speeds increase with both $H$ and the reference depth, the largest proportionate increase being in $c_{1}$, which ranges from 2.57 to $3.02 \mathrm{~m} \mathrm{~s}^{-1} ; c_{6}$ ranges from 0.45 to $0.56 \mathrm{~m} \mathrm{~s}^{-1}$. It is not clear which choice of reference depth and $H$ is best. From (34), the natural frequencies $\omega$ are proportional to the square root of $c_{m}$, so the choice of $H$ and reference depth can change the natural frequency for mode 1 by up to $10 \%$ and for mode 6 by up to $5 \%$.

Figures $5 \mathrm{~b}$ and $5 \mathrm{c}$ illustrate the dependence of $c_{m}$ on $H$ and the reference level used for $\sigma$ for the Atlantic and Indian Oceans. Comparing Fig. 5a to Fig. 5c, we see that the phase speeds for the full Indian and Pacific Oceans are generally quite similar while the phase speeds for $m \leq 2$ in the Atlantic are slower. The variation of the phase speeds with $H, \sigma_{0}$ and $\sigma_{2}$ in each of the three full basins is comparable. In all the figures of this paper-other than Figs. 5a-c-we use $H=4191 \mathrm{~m}$ and $\sigma_{2}$ as "standard" values. We occasionally comment on results obtained using alternative choices. 
Figure 5d displays values calculated using that choice for two months (April and October) and three regions (the full Pacific, its eastern and its western halves). The phase speeds are smaller in the west Pacific than the east Pacific, $c_{1}$ in April varying from $2.68 \mathrm{~m} \mathrm{~s}^{-1}$ in the west to $3.19 \mathrm{~m} \mathrm{~s}^{-1}$ in the east Pacific. The seasonal variation in $c_{m}$ for the full Pacific is of order $2 \%$ for $c_{1}$ and $1 \%$ for $c_{6}$ whereas it is of order $10 \%$ for $c_{1}$ in the west Pacific. The seasonal variations in $c_{m}$ for the full Atlantic and Indian Oceans are of similar magnitudes to those for the full Pacific. It would be possible to calculate the vertical modes using the correct monthly mean values but the impact on the results would be smaller than variations that could arise from longitudinal variations in the region where the oscillations have maximum magnitude. In all figures of this paper other than Fig. 5d we have used April monthly mean data to calculate $c_{m}$.

Figure $6 \mathrm{~b}$ displays the structure of the first six normalized vertical modes, $\bar{p}_{m}(z)$, for our standard choices (April $\sigma_{2}$ potential densities with $H=4191 \mathrm{~m}$ ) in the Pacific. We note that the first baroclinic mode is surface intensified and that the other modes also resemble the product of $\cos (m \pi \bar{z})$ and an envelope profile that reduces in amplitude with depth. This is because, taking $\lambda$ to be a Lagrange multiplier, the vertical modes minimize

$$
J \equiv \int_{-1}^{0}\left[\frac{1}{N^{2}}\left(\frac{d \bar{p}}{d \bar{z}}\right)^{2}-\lambda \bar{p}^{2}\right] d \bar{z}
$$

for functions that satisfy the same boundary conditions as the vertical modes. The values of $N^{-2}$ increase markedly with depth below the thermocline and variations in $d \bar{p} / d \bar{z}$ in the vertical modes are increasingly suppressed below the thermocline as depth increases.

The structure of the first six meridional modes, $\tilde{\phi}(y)$, as a function of latitude for the first baroclinic mode is displayed in Fig. 6a. Mode $n=0$ has a simple Gaussian shape and is largely confined to within $5^{\circ}$ of the equator. The higher lateral modes are much less tightly confined to the equator. As illustrated in Part I, the meridional modes for higher vertical modes are more tightly confined to the equator than those for the $m=1$ mode. Figures $6 \mathrm{c}$ and $6 \mathrm{~d}$ illustrate the two-dimensional structure of modes $(1,0)$ and $(2,1)$, respectively. One can see that quite complex spatial patterns can be constructed from linear combinations of even fairly low numbered meridional and vertical modes.

\section{c. Dependence of solutions on the kind and type of simulation}

Figure 7a shows how $S_{T}$ (the fraction of the variance not captured by the idealized simulations, defined in section $3 \mathrm{~g}$ and Table 1) depends on the four types of forcing and the three kinds of simulations (described in section $3 f$ and Table 1 ) for the first two vertical and meridional modes (four modes in all) in the Pacific. For each mode, $S_{T}$ is plotted for the four types of forcing, $A$ to $D$, from left to right. No forcing (type $A$ ) using the natural frequency (kind $N$ ) gives $S_{T}$ greater than 0.35 in all four cases. Even with no forcing the $H$ and $F$ simulations give $S_{T}<$ 0.1 for all modes. For the natural solutions (black dots), in all four cases the full forcing, type $D$, gives the lowest value of $S_{T}$ and this value is comparable with that obtained using the $H$ and $F$ simulations with no forcing. The $D_{F}$ simulation also has the smallest value of $S_{T}$ in all cases though $D_{F}$ is only clearly better than $C_{F}$ for the $(2,0)$ mode. The $B_{N}$ simulations forced just by winds perform better (worse) for $n=0(n=1)$ than the $C_{N}$ simulations that include baroclinic pressure forcing.

The forcing of the $\tilde{v}_{m, n}$ solutions by the zonal wind stresses and the full pressures forces on the western and eastern boundaries can be calculated using (22), (32), and (33). These time series for modes $(1,0)$ and $(1,1)$ in the Pacific are plotted in Fig. 8. For mode $(1,0)$ the full pressure forces on the boundaries are almost negligible. For mode $(1,1)$ the full pressure forces vary less rapidly in time than the zonal wind forcing but are not negligible, and slow variations in them appear to partially compensate slow variations in the zonal wind forcing. Slow variations in forcing can be largely captured in the least squares fit solutions by variation of the amplitude of $\alpha_{5}$ in (38). The relative impact of variations in the pressure forcing and the wind forcing within a time integration period on the quality of the solutions can be assessed by comparing the residuals for the least squares simulations $A_{H}, B_{H}$, and $D_{H}$ in which the amplitudes of the forcings are held fixed. Figure $7 \mathrm{a}$ shows that for these solutions (the red crosses), the improvement in fit between simulations $A_{H}$ and $B_{H}$, due to including the wind forcing, is significantly greater than that between simulations $B_{H}$ and $D_{H}$, due to including the pressure forcing. This is true for all modes with $m \leq 4$ and $n \leq 3$ (not shown).

The time series of $d \tilde{Y}_{m, n} / d t$ for these modes are also plotted as gray lines on the same figures because they also drive the ODE for $v_{m, n}$, (33). These time series are evidently very noisy and appear to play a minor role in driving longer period variations in $v_{m, n}$. This noise in the spline representation of $d Y_{m, n} / d t$ contributes noise to $d^{2} \tilde{\boldsymbol{v}} / d t^{2}$ and limits its accuracy. This is one of the two main reasons why our presentation focuses on $S_{T}$ (rather than $S_{A}$ ) as a measure of the quality of the simulation. The second reason for focusing on $S_{T}$ is that it is a more direct overall measure of the quality of the time series fit. An alternative method to splines for the calculation of $d \tilde{Y}_{m, n} / d t$ could give less noisy calculations of this term and either improve the least squares fit solutions or at least make their results easier to interpret.

It is perhaps difficult to gauge from these figures how convincing the agreement really is. For this reason we mention that at one stage we corrected the $\exp \left(-\tilde{y}_{m}^{2} / 4\right)$ term in (28) that had previously read $\exp \left(-\tilde{y}_{m}^{2} / 2\right)$. This reduced the value of $S_{T}$ in the $N$ and $H$ kinds of simulations for most of the leading modes by a factor of 2-3. The least squares method was a useful tool for finding errors and improving the results.

Figure $7 \mathrm{~b}$ indicates how the best fit parameters, $\alpha_{3}$ and $\alpha_{2}$, multiplying the forces along the $x$ and $y$ axes in simulation $D_{F}$ compare with the value of 1 expected from the "physical" theory. The 20 segments are used to calculate a mean value, $\mu$, for each parameter. The standard deviation of this mean, $\sigma_{m}$, is calculated from the standard deviation of the parameter values, $\sigma_{p}$, assuming that the samples are independent: $\sigma_{m}=\sigma_{p} / \sqrt{19}$. The mean values, $\mu$ (full lines) and $\mu \pm 2 \sigma_{m}$ (dashed lines) are presented in the figure. The 
a)

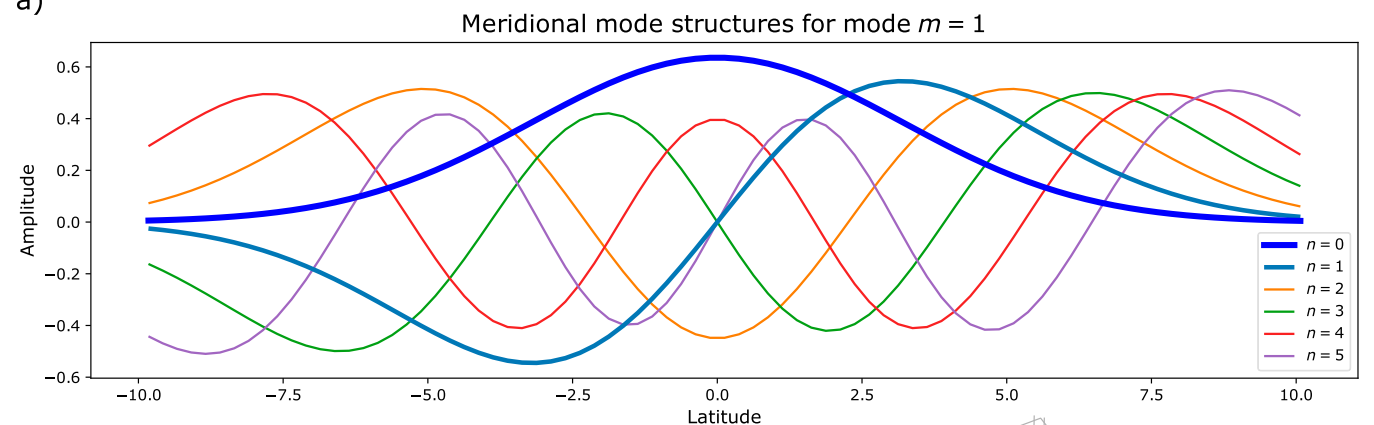

b)

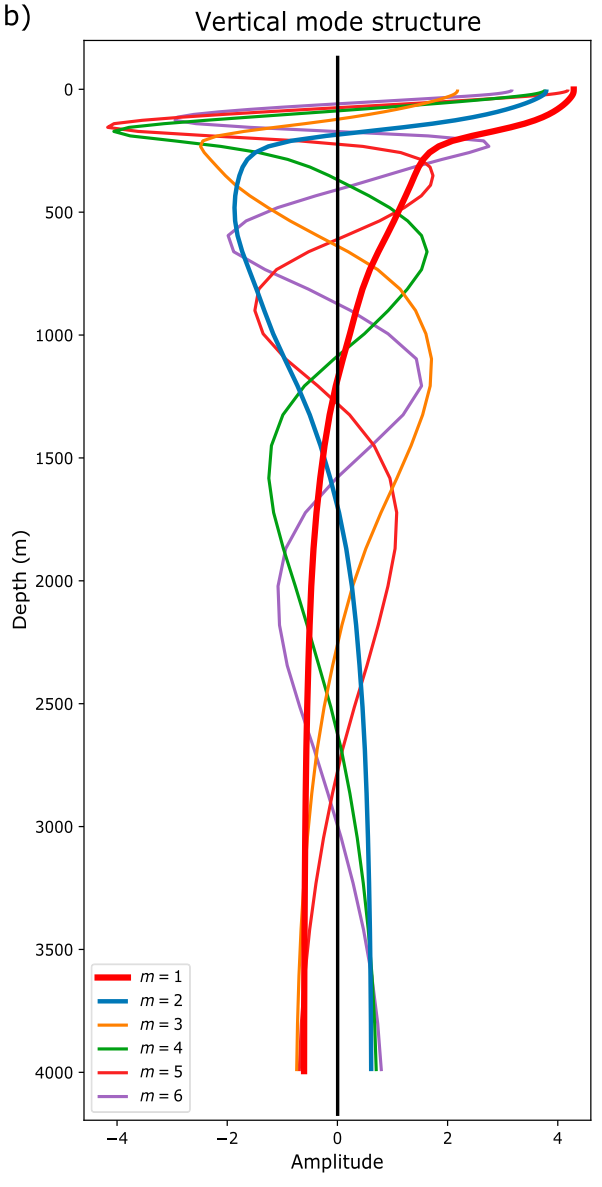

c)

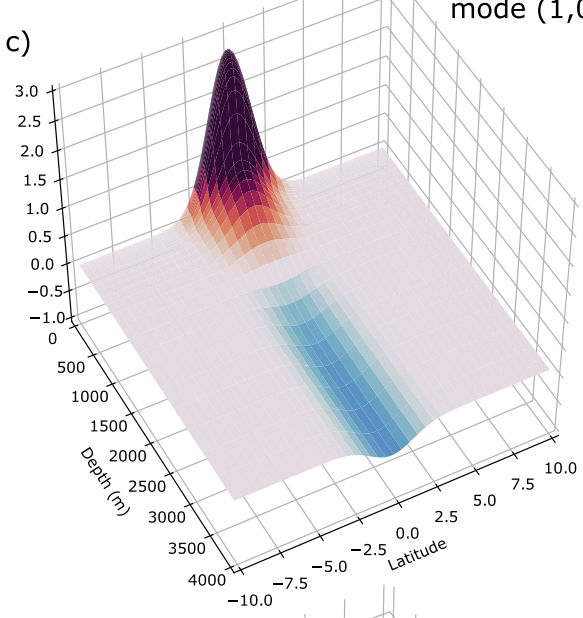

d)

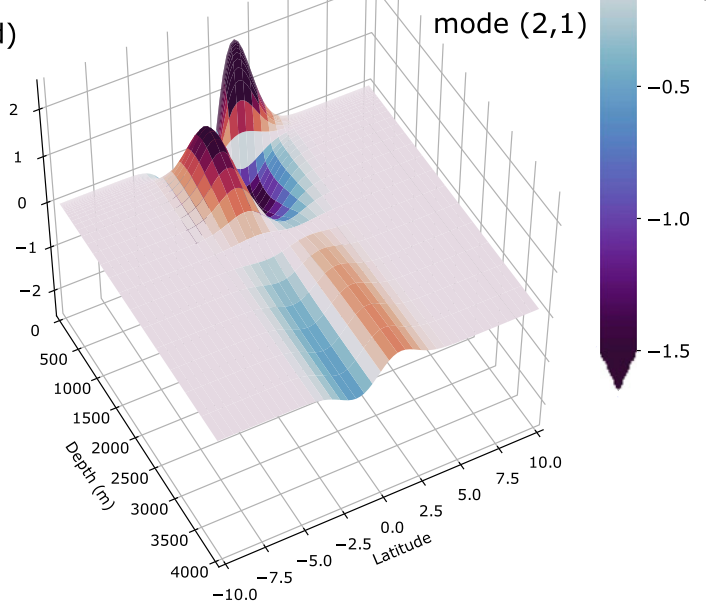

FIG. 6. The amplitudes of the first six nondimensional modes for the Pacific: (a) the meridional modes $\phi_{1, n}(y)$ for the first baroclinic mode as a function of latitude, (b) baroclinic vertical modes $\hat{p}_{m}(z)$ as function of dimensional depth $z$, (c) 3D projection showing the depth-latitude structure of mode $(1,0)$, which corresponds to the thick red curve in (b) $(m=1)$ and the thick blue curve in (a) $(n=0)$, and (d) 3D projection showing the depth-latitude structure of mode $(2,1)$, as in (c), but with $m=2$ and $n=1$.

parameters for the force along the $x$ axis (red lines) have a fairly large standard deviation so the departure of the mean from the expected value of 1 does not appear to be particularly significant. The parameter multiplying the meridional forcing has a much smaller standard deviation. Although the mean value of this parameter (blue line) lies fairly close to 1 , between
1.1 and 1.15 , it appears to be significantly larger than 1. Simulations with $H_{M}=25 \mathrm{~m}$ have mean values for this parameter in the same range while those with $H_{M}=100 \mathrm{~m}$ have larger values. So the reason for this discrepancy is unclear.

Figure $7 \mathrm{c}$ indicates how the squared frequencies $\omega^{2}$ selected by the least squares fit in simulation $D_{F}$ compare with the 

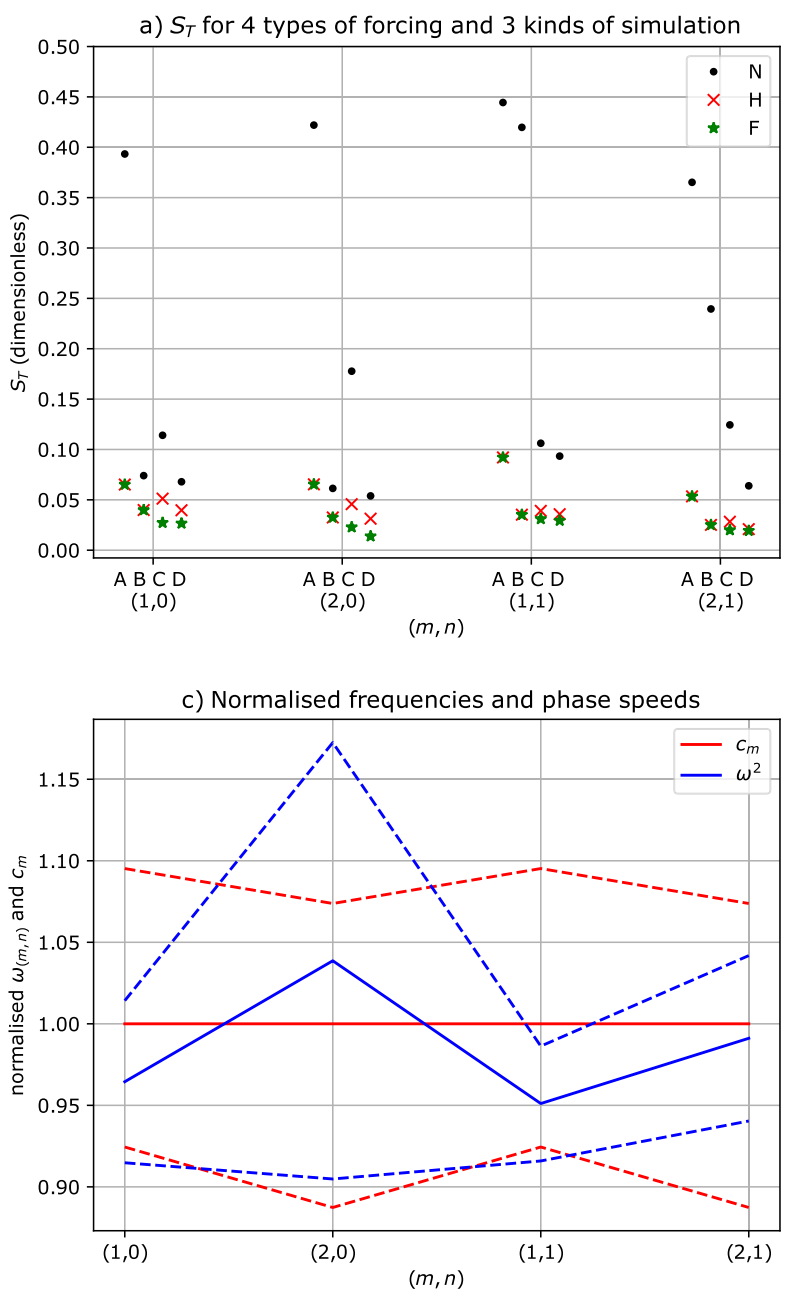

values, $\omega_{m, n}^{2}$, predicted by the theory. The mean and estimated uncertainty of $\omega^{2} / \omega_{m, n}^{2}$ in simulation $D_{F}$, calculated as in Fig. 7b, are presented as the blue lines in Fig. 7c. The mean squared frequencies for the least squares fit are consistent with the theory (with our choice of $H$ and $\sigma_{2}$ ). The red dashed lines display the ratios $c_{m}(\mathrm{EP}) / c_{m}(P)$ and $c_{m}(\mathrm{WP}) / c_{m}(P)$, where EP, $\mathrm{WP}$, and $P$ denote the east Pacific, west Pacific, and full Pacific regions and the phase speeds have been calculated using the April data. A solid red line plots $c_{m}(P) / c_{m}(P)=1$. The least squares fit frequencies appear to be comparable with the range of variation expected from the variation in density stratification.

\section{d. A survey of the quality of the solutions}

Before assessing the quality of the simulations for a wider range of modes, it is useful to note how the variance $V_{T}$ in the modes varies with the vertical and meridional wavenumbers. Figure 9a presents $V_{T}$ for the first five vertical and meridional modes in the Pacific calculated by projecting the NEMO meridional velocities directly onto them. Although the lowest meridional and vertical modes clearly have the largest variances, the variances of these modes decrease fairly slowly with vertical and meridional wavenumber. In the case of the vertical

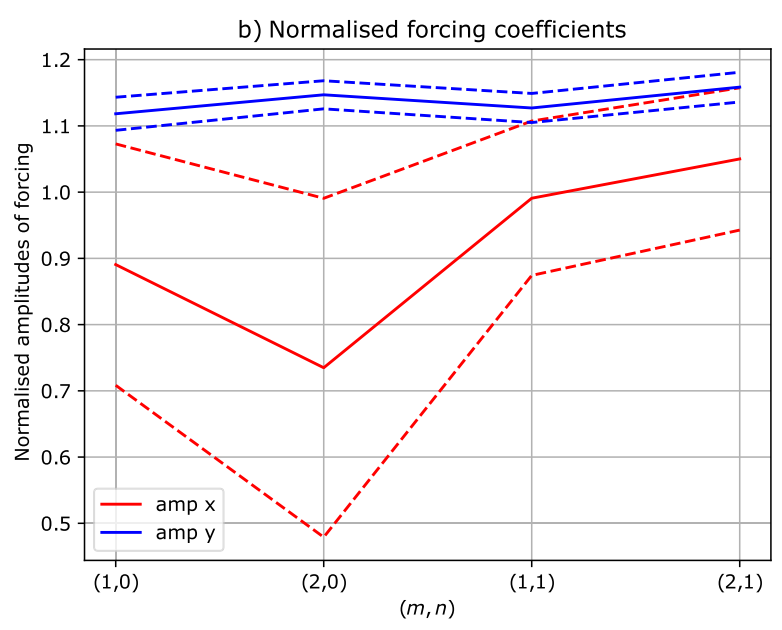

FIG. 7. (a) The $S_{T}$ (i.e., the fraction of the variance not captured by the idealized simulations) for three kinds of simulation (see labels) and four types of forcing, from $A$ (no forcing) on the left to $D$ (full forcing) on the right. Also shown are the mean (full lines) and the mean \pm 2 standard deviations (dashed lines) of (b) the amplitudes multiplying the wind stresses along the $x$ (blue) and $y$ (red) axes and (c) $\omega^{2} / \omega_{m, n}^{2}$ (blue) obtained in simulation $D_{F}$. In (c), the red dashed lines display the ratios $c_{m}(\mathrm{EP}) / c_{m}(P)$ and $c_{m}(\mathrm{WP}) / c_{m}(P)$, where EP, WP, and $P$ denote the east Pacific, west Pacific, and full Pacific regions, and the full red line is $c_{m}(P) / c_{m}(P)=1$. All panels show results for the first four modes $(m, n)$ in the Pacific with $m \leq 2$ and $n \leq 1$.

modes this is not surprising because none of these modes varies greatly within a mixed layer that is $50 \mathrm{~m}$ deep.

Figure $9 \mathrm{~b}$ presents $S_{T}$ for the same set of modes calculated by simulations $D_{N}$ (solid lines) and $D_{F}$ (dashed lines). For the first four vertical and meridional modes all (sixteen) $D_{F}$ solutions have $S_{T}<0.12$. For the first two (three) vertical and meridional modes all $D_{N}$ solutions have $S_{T}<0.09\left(S_{T}<0.15\right)$. The values of $S_{T}$ for the higher modes are larger but this is partly because the variance of these modes is smaller (Fig. 9a); the largest values of $V_{T} S_{T}$ are in fact obtained for modes with $m=1$ or $m=2$ (not shown).

Simulations using $H=5120 \mathrm{~m}$ and $\sigma_{0}$ give smaller values of $S_{T}$ for the first and second baroclinic modes but larger values of $S_{T}$ for the higher vertical modes (not shown). Indeed the values for $S_{T}$ for the $D_{N}$ solutions with $m=1$ and $2 \leq n \leq 4$ are half those obtained using $H=4191 \mathrm{~m}$ and $\sigma_{2}$. The improved performance for the first baroclinic mode may be because it is near-surface intensified. The best solutions for these modes are obtained using the largest value of $H=5596 \mathrm{~m}$ that is common to all latitudes [in other words, the smallest value of $H_{\max }(y)$ ].

Figures $9 \mathrm{c}$ and $9 \mathrm{~d}$ present $S_{T}(m, n)$ for the Atlantic and Indian Oceans, respectively. Comparing them with Fig. $9 \mathrm{~b}$ for 

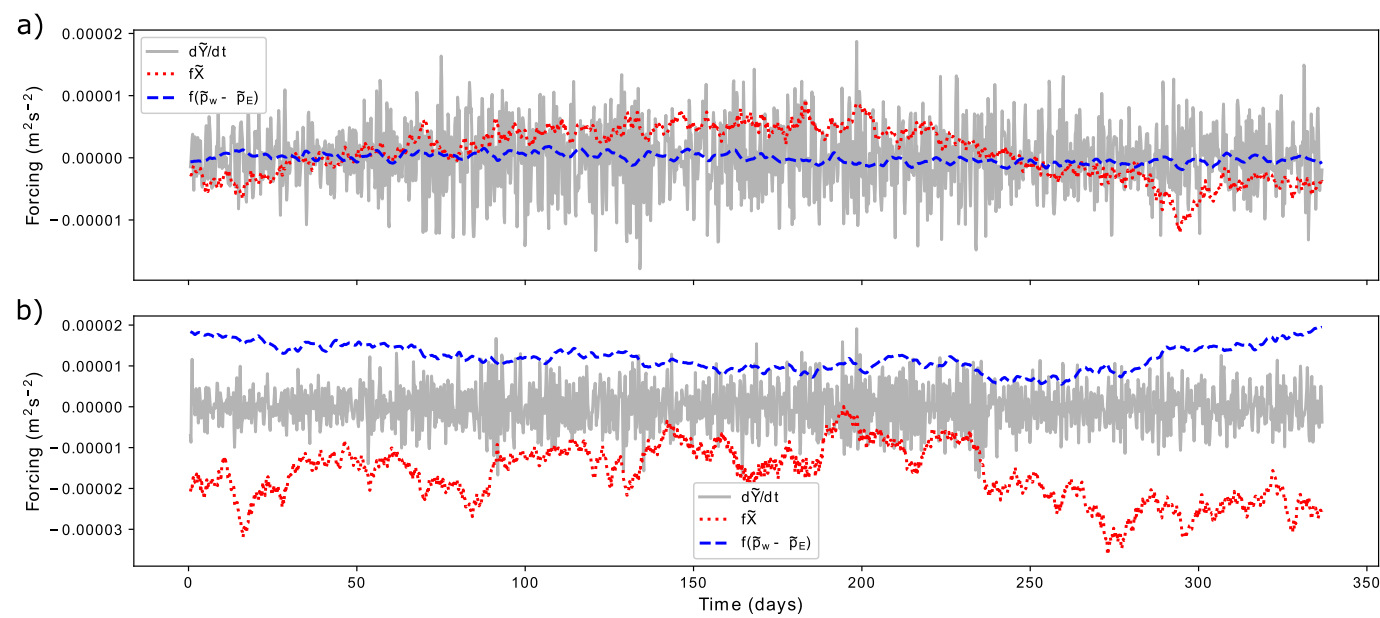

FIG. 8. Time series for the Pacific of the forcing $\tilde{X}_{m, n}^{f}$ by the zonal winds (red), $g \Delta \tilde{h}_{m, n}^{f}$ by pressures on the boundaries (blue), and $d \tilde{Y}_{m, n} / d t$ by the meridional winds (black) for modes with $(m, n)$ equal to (a) $(1,0)$ and (b) $(1,1)$.

the Pacific, one sees that the simulations in the Indian Ocean are generally somewhat better and those in the Atlantic somewhat worse than those in the Pacific. The $D_{N}$ simulations with $m=5$ and $n=1$ in the Atlantic, $n=1$ or $n=3$ in the
Pacific Ocean and $n=0$ or $n=2$ in the Indian Ocean have relatively large values of $S_{T}$. Mode $(5,3)$ in the Pacific has $S_{T}=$ 0.425 and 0.134 for simulations $D_{N}$ and $D_{F}$, respectively, and is one of the most poorly simulated modes. The time series for
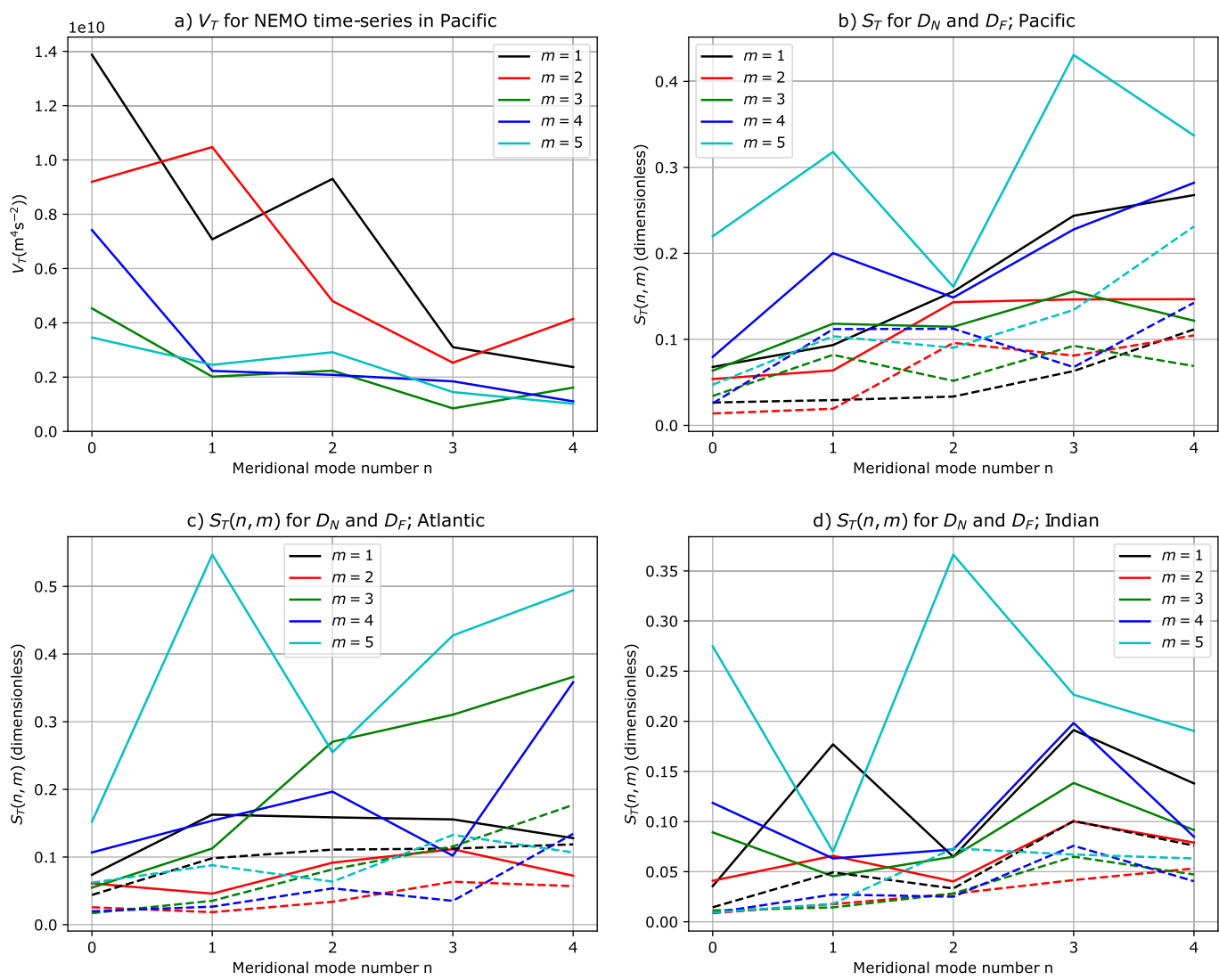

FIG. 9. (a) The $V_{T}$ for the first five meridional modes (abscissa) and the first five vertical modes (see the labels) calculated by projecting the NEMO Pacific meridional velocities directly onto the modes. Also shown is $S_{T}$ for the same modes in simulations $D_{N}$ (full lines) and $D_{F}$ (dashed lines) for the (b) Pacific, (c) Atlantic, and (d) Indian Oceans. 

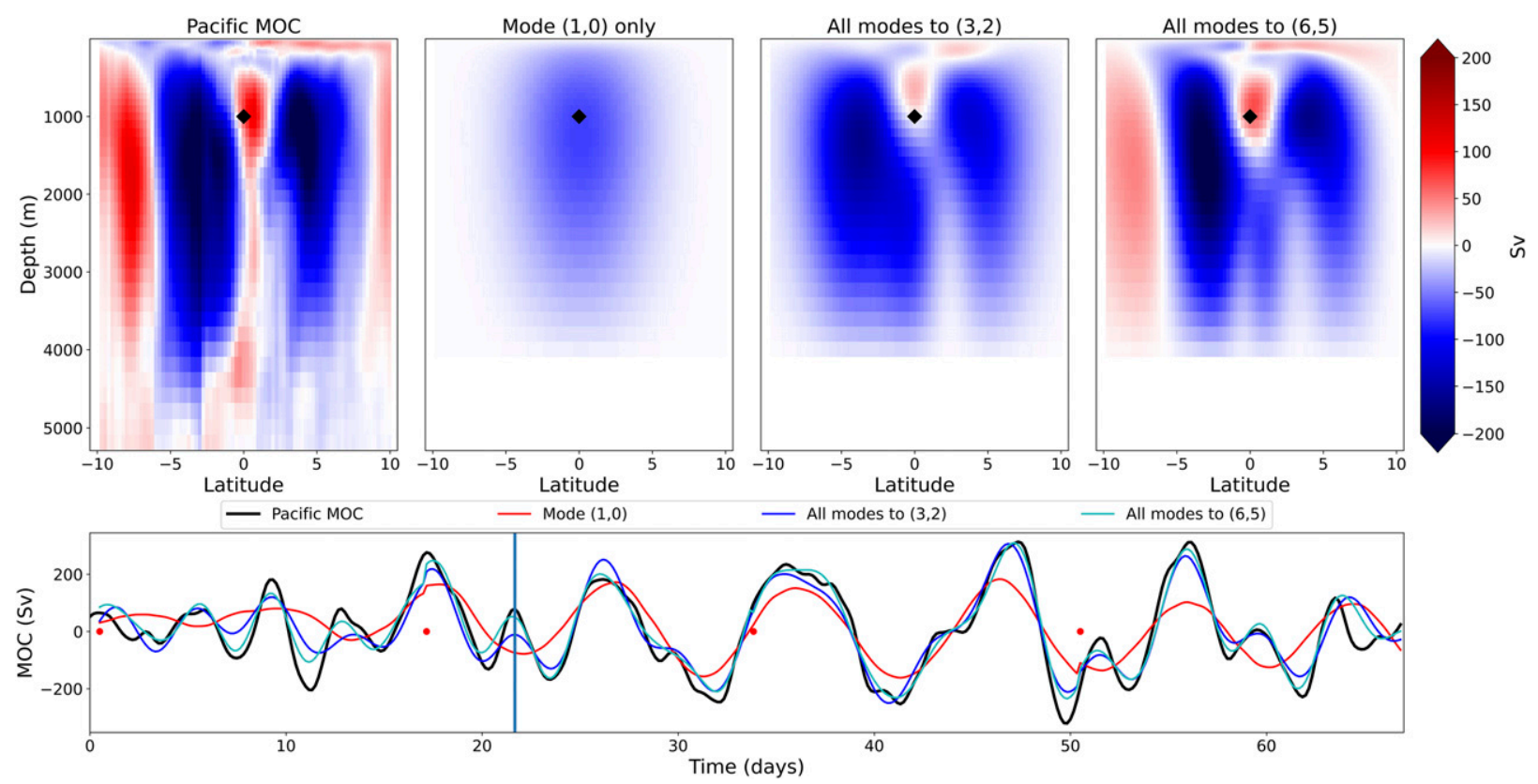

FIG. 10. (top) MOC streamfunction for the Pacific Ocean as a function of latitude and depth on day 22 (time step 130) (left) calculated directly from the NEMO integration, and reconstructed from a simulation of type $D_{H}$ with $H=5495 \mathrm{~m}$ using (left center) mode (1,0) only, (right center) all modes with $m \leq 3$ and $n \leq 2$, and (right) all modes with $m \leq 6$ and $n \leq 5$. (bottom) Time series of the MOC streamfunction for the Pacific on the equator at 1583-m depth for the NEMO integration (black), mode (1,0) only (red), all modes with $m \leq 3$ and $n \leq 2$ (bright blue), and all modes with $m \leq 6$ and $n \leq 5$ (green/blue).

$D_{N}$ and $D_{H}$ are illustrated in Fig. 4b. Simulation $D_{N}$ (red line) reproduces the modes quite well at times (e.g., from days 230 to 290 ) but poorly at others (e.g., from days 110 to 130). It appears from Fig. $4 \mathrm{~b}$ that the simulation of this mode is poor mainly because it fails to follow the slow variations in the amplitude accurately. This also appears to be the case for the other poorly simulated modes noted above.

Similarly (as alluded to earlier) the time series for $(m, n)=$ $(2,2)$ in the Pacific plotted in Fig. 4a has the largest value of $S_{T}$ (and is in this sense the most poorly simulated) for both $D_{N}$ and $D_{F}$ of all time series for the Pacific with $1 \leq m \leq 3$ and $0 \leq n \leq 2$ (i.e., the first 9 modes). Careful examination of Fig. 4a and of the corresponding slow solution (not shown) shows that the slow variations in the $(2,2)$ mode in the Pacific are also not accurately represented by the $D_{N}$ or the slow solution.

Particularly for the natural solutions, the statistical fit of the time series $S_{T}$ for integration periods of $800 \mathrm{~h}$ is less good than those for the $400 \mathrm{~h}$ integration periods just discussed. As mentioned in section $3 \mathrm{~g}$, a measure of the quality of the simulation of $d^{2} \tilde{\boldsymbol{v}} / d t^{2}$ has also been calculated. This measure has some merit in that it is much less sensitive to the length of the segment used in the calculations. For the segment length presented the results for this measure (not shown) are qualitatively similar to those already presented. The pros and cons of the two measures are discussed further in section $4 \mathrm{f}$.

\section{e. Projection onto $M O C$}

The $\tilde{v}_{m, n}$ modes described above combine to generate quite complex patterns in the MOC streamfunction. The upper-left panel of Fig. 10 illustrates the MOC streamfunction at a single time as a function of latitude and depth and the panels to its right show reconstructions using the $D_{H}$ simulations progressively including more vertical and meridional modes. As more modes are included the reconstruction captures more of the complex meridional structure of the streamfunction. The lower panel, which shows time series of the same reconstructions and NEMO integration on the equator at 1583-m depth, shows that the richer modal reconstructions are required to capture the full variation of the peaks and troughs in the MOC at the equator. An animation of the information presented in Fig. 10 shows clearly how the addition of higher vertical and meridional modes to the solution results in an increasingly realistic representation of the MOC variability at all depths and latitudes (see Fig. S1 in the online supplemental material). Part I presents alternative sections through these $3 \mathrm{D}$ reconstructions and statistical analyses that confirm that, as the number of meridional modes used in the reconstruction increases, the fraction of the NEMO MOC accounted for by the linearized simulations increases, particularly at higher latitudes and greater depths.

\section{f. Slowly varying solutions in the Pacific}

We now investigate the slow solutions, described in section 3e, obtained for the Pacific by applying to $\tilde{v}_{m, n}$ calculated using (41) a running mean whose length is the period of the natural frequency of the mode. The red dashed lines in Fig. 11 show these slow modes for $(m, n)$ equal to $(1,0)$ in Fig. 11a and $(1,1)$ in Fig. 11b. The black solid lines are the time series calculated by applying the same time filter to the amplitudes calculated directly from the NEMO velocities. In both cases the 

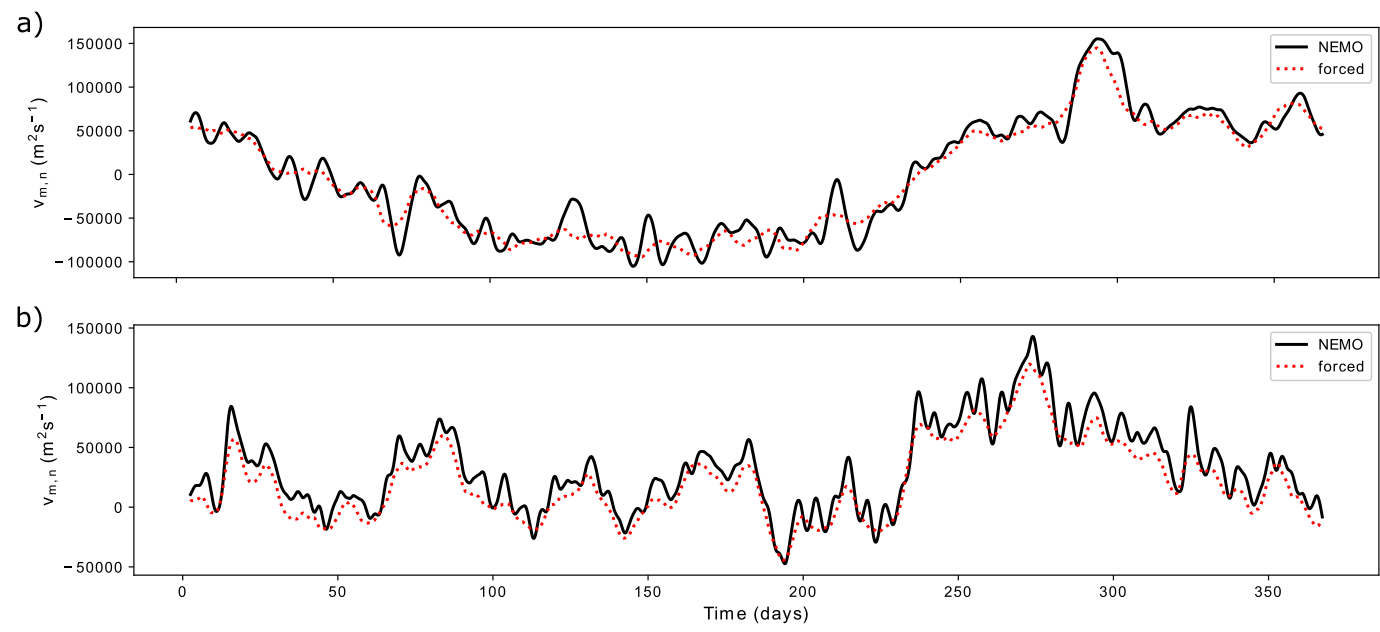

FIG. 11. Time series for the Pacific obtained by applying a running mean, of length equal to the period of the resonant frequency, to the amplitude of modes obtained directly from the NEMO $\tilde{v}_{m, n}$ velocities (black solid line) and from the slow solution obtained from (41) (red dashed line) for (a) mode (1,0) and (b) mode $(1,1)$.

agreement is good, particularly on longer time scales; the black lines have residual amplitudes at fairly high frequencies. Figure 12a displays the mean square values of these filtered NEMO $\tilde{v}_{m, n}$ values (the black lines in Fig. 11) and the mean square values of the difference between them and the slow mode solutions (the difference between the black and red lines in Fig. 11). The normalized residuals (the ratio of the dashed and the solid lines) are small for $m \leq 2$ and $n \leq 4$ but increase rapidly for larger values of $m$ (particularly for even values of $n$ ).

The red and blue lines in Fig. 8 show respectively the wind $\tilde{X}_{m, n}^{f}$ and pressure $\Delta \tilde{h}_{m, n}^{f}$ terms that contribute to the numerator on the rhs of (41) for the same modes as presented in Fig. 11.
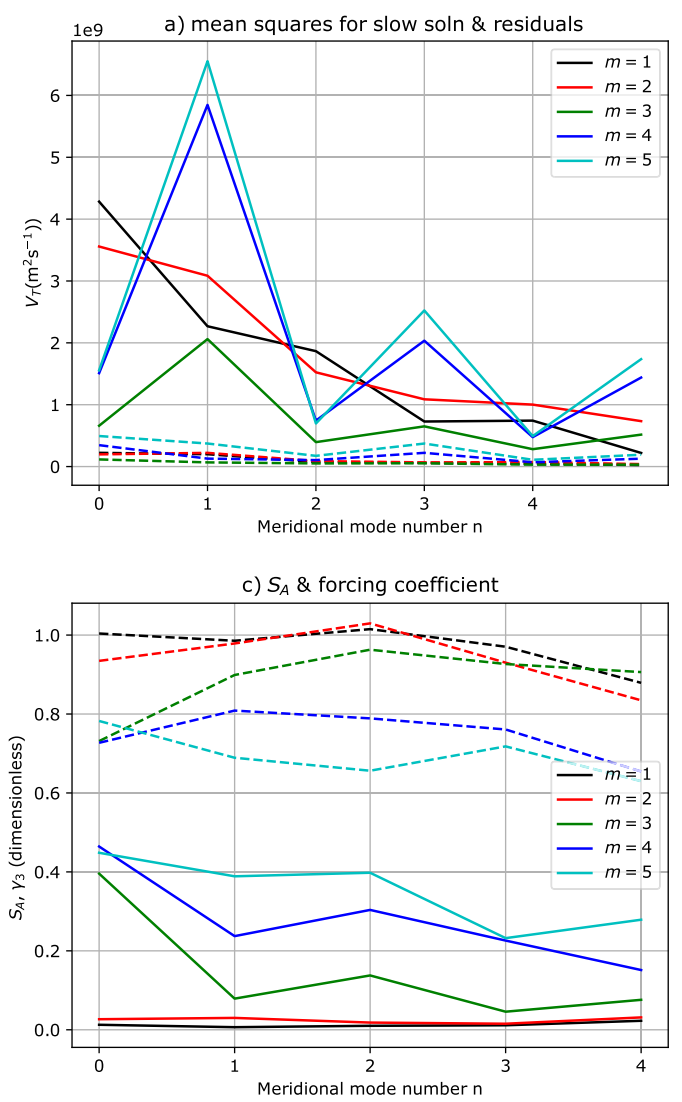

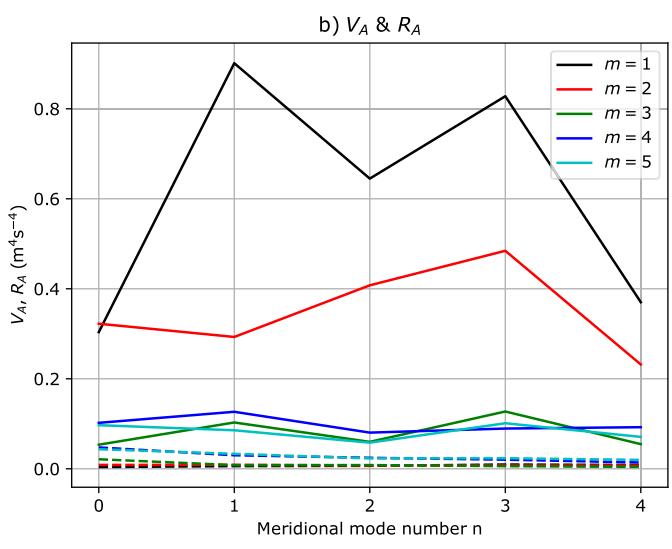

FIG. 12. (a) Mean square values of running means of NEMO modes (full lines) and their mean square differences from the slow $\tilde{v}_{m, n}$ solutions for the Pacific given by (41) (dashed lines). (b) The $V_{A}$ (solid) and $R_{A}$ (dashed), and (c) $S_{A}$ (solid) and $\gamma_{3}$ (dashed) for $D_{F}$ simulations of $\tilde{u}_{m, n}$ in the Pacific. 

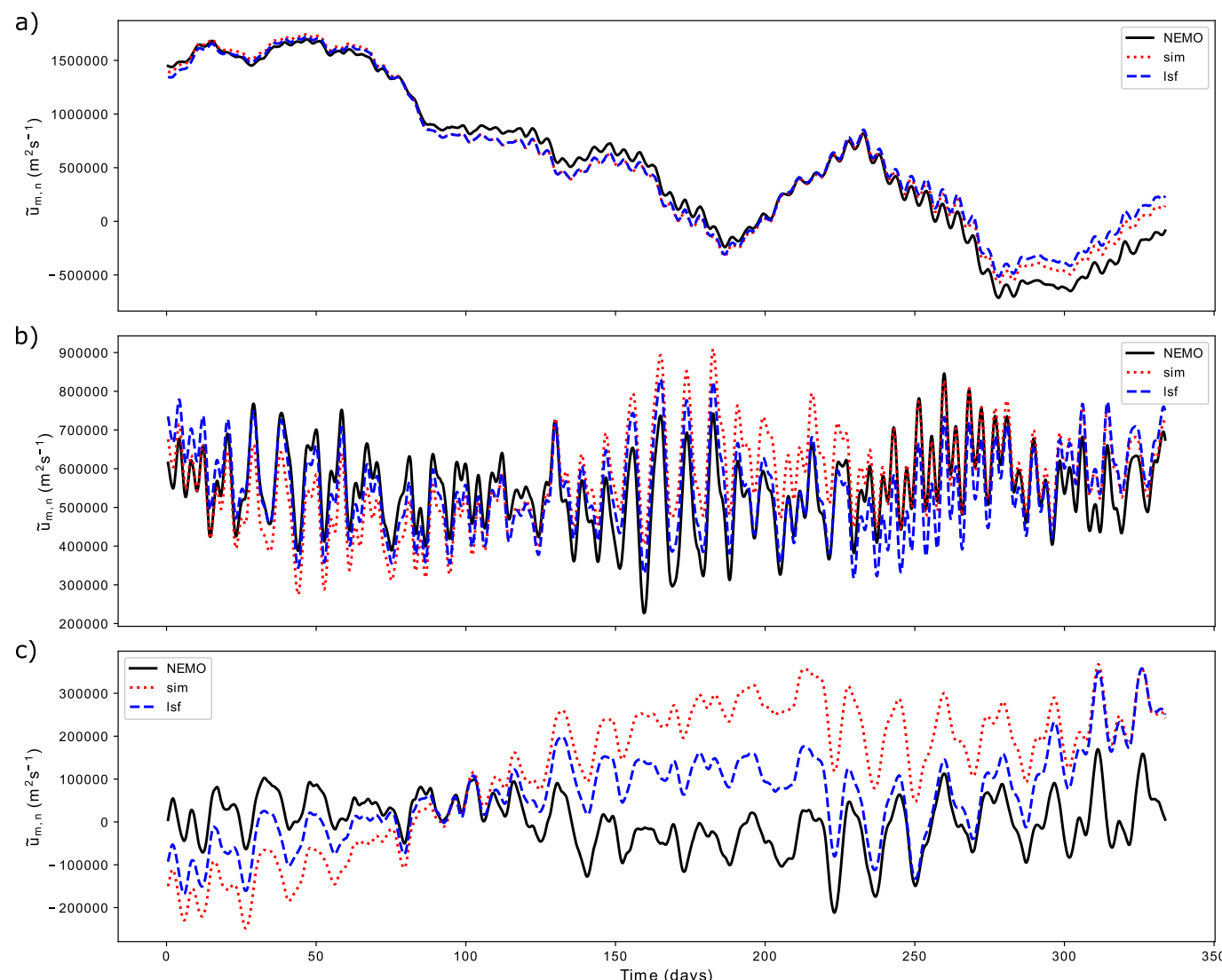

FIG. 13. Time series for the Pacific of (a) $\tilde{u}_{1,0}$, (b) $\tilde{u}_{1,1}$, and (c) $\tilde{u}_{3,1}$. The full black lines are calculated directly from NEMO data. The red dotted and blue dashed lines are simulations of types $D_{N}$ and $D_{F}$, respectively.

For mode $(1,0)$ the pressure term makes only a very small contribution but for mode $(1,1)$ its contribution is important. Neglecting it would give a very poor simulation of the slowly varying solution for $\tilde{\boldsymbol{v}}_{m, n}$.

\section{g. Solutions for the zonal velocities in the Pacific}

Figure 13 presents time series of the zonally integrated zonal velocities $\tilde{u}_{m, n}$ for modes $(1,0),(1,1)$, and $(3,1)$ in the Pacific. The linearized simulations have been calculated as described in the last paragraphs of sections $2 \mathrm{~d}$ and $3 \mathrm{f}$. The solutions for modes $(1,0)$ and $(1,1)$ are remarkably different; the highfrequency variations in mode $(1,1)$ are much larger than those in mode $(1,0)$ and the reverse is true for the low-frequency variations. In both cases, both the least squares fit simulations, $D_{F}$, and the natural simulations, $D_{N}$, generally capture both the short-period and slower variations rather well. Mode $(3,1)$ has small-amplitude, short-period variations that are also well captured but the two simulations of the slower variations are both poor. Figures $12 \mathrm{~b}$ and $12 \mathrm{c}$ summarize the dependence of the quality of the solutions on the meridional- and verticalmode numbers. Figure $12 \mathrm{~b}$ shows that the mean value of $(d \tilde{u} / d t)^{2}\left(V_{A}\right.$, solid lines) is largest in vertical modes 1 and 2 and that the residual $R_{A}$ is smallest in these modes. Figure $12 \mathrm{c}$ shows that the ratio $R_{A} / V_{A}\left(S_{A}\right.$, solid lines) increases rapidly with vertical-mode number and increases slightly as the meridional-mode number decreases. It also shows that the least squares fit coefficient $\gamma_{3}$ multiplying $\tilde{X}_{m, n}+\Delta \tilde{h}_{m, n}$ (dashed lines) is close to its expected value of 1 for $m \leq 2$ and $n \leq 2$ and generally is inaccurate for other values of $m$. As discussed in the next section, further work would be required to relate these results to variations in the equatorial undercurrent and the equatorial countercurrents.

\section{Concluding summary and discussion}

The large-amplitude, high-frequency oscillations in the zonally integrated meridional circulations near the equator, that are found in the Pacific, Indian, and Atlantic Oceans in NEMO simulations and described in some detail in the first part of this study, have been shown to arise from the projection of the zonally integrated surface wind and bottom pressure forcing onto the vertical and meridional (equatorially trapped) normal modes of motion. For the first few meridional and vertical modes, it has been shown that the frequencies of the oscillations and amplitude of the response can be well simulated even though the horizontal variations in the stratification and time-mean currents are neglected. It is shown in Part I, using the calculations explained in this part of the paper, that the high-frequency variations in the MOC near the equator can be accurately reproduced in the top 2000 (3000) $\mathrm{m}$ within 
$5^{\circ}\left(7.5^{\circ}\right)$ using only $3(6)$ vertical and meridional modes. Slower variations in modes (with periods exceeding 10 days) with vertical- and meridional-mode numbers $m \leq 2$ and $n \leq 4$ have also been shown to be well reproduced by "quasi-stationary" solutions for these modes. The accompanying variations in the zonal velocities have been shown to be well captured for the first and second baroclinic vertical modes (at least for the first three meridional modes) but poorly represented for higher vertical modes.

It is worth noting that we have used "classical" baroclinic modes in our calculations rather than the surface modes proposed by LaCasce (2017). We have repeated our calculations using the surface modes' boundary condition, $\left\langle p^{\prime}\right\rangle=0$ rather than $\left\langle w^{\prime}\right\rangle=0$ at $z=-H$, and find that this roughly doubles the size of the residuals between the idealized and the NEMO simulations. We suggest that two reasons may account for this. First, our solutions do not require the ocean bottom to be flat but only that $H_{\max }(y)$ (the maximum bathymetric depth as a function of $y$ ) be independent of $y$. Second, we suggest that a sufficiently good approximation to the bathymetry that constrains the motions can be specified for which this is true (noting that we obtain our best results using a maximum bathymetry that disregards the deep-ocean trenches).

It is conceivable that the fluctuations studied in this paper are more damped in reality than they are in the NEMO integrations. The fact that Farrar and Durland (2012) found significant power in TAO mooring data in long zonal wavelength modes of similar periods to the Yanai and internal gravity wave modes that we have simulated in this study gives some reassurance that the fluctuations occur in reality. But suitable quantitative comparison between the TAO data, the NEMO integrations, and the idealized simulations are needed to give further evidence on whether the fluctuations in the MOC simulated in this paper are indeed of realistic amplitude.

The relationship of the slow solutions to theories of the equatorial undercurrent, summarized for example by Vallis (2017), may also be worth further study. The use of (41) as a simplified balance equation for the meridional flow is very attractive, but the separation of the specification of the boundary pressure forces from the solution is an obvious limitation in this context and the convergence of the slow modes expansion may need careful consideration. It would also be interesting to investigate the slowly varying vertical velocities associated with the normal modes. These vertical velocities penetrate deep into the water column and could play an important role in the upwelling of water from mid-depths into the thermocline or near surface waters.

The limited skill of the higher vertical and meridional modes found in this study also applies to similar analyses of lowwavenumber Rossby-Haurwitz waves in the atmosphere (Madden 1979). In the atmosphere, however, the agreement of the phase speeds is usually considered to be limited to the modes of lowest wavenumber because only those modes have fast enough phase speeds for the zonal wind velocities to be negligible. In our case it does not make sense to superimpose a zonal flow on the solutions because the zonal flow would not satisfy the boundary conditions but it is plausible that the higher vertical and meridional modes interact more with the zonal flows, particularly in critical layers. Another problem with the simulation of the higher modes may be that the projection onto them assuming a "fixed" mixed layer depth of $50 \mathrm{~m}$ is not accurate enough; projection of the wind stresses in the vertical using a more accurate (spatially and temporally varying) representation of the mixing layer depth for momentum may give better results. Use of the local time-mean stratification rather than a "global" average, in the manner of a WKBJ approximation, may also possibly give better results. One might also need to use normal modes that are calculated consistently with the numerics of the NEMO model (Thuburn and Woollings 2005).

The key role played by oceanic inertia-gravity and Yanai waves in the response of the zonally integrated circulations to the wind forcing, raises obvious questions about their role in fluxing momentum input by variable surface winds into the ocean interior. Large-amplitude, relatively low-frequency inertia-gravity waves could be generated by (fluctuating) wind stresses along the equator. "Shear-spiking" events (Crawford and Large 1996; Jochum et al. 2013) could generate downward propagating waves carrying westward momentum. These waves could contribute to the generation of the vertically stacked zonal jets and basin modes discussed by Greatbatch et al. (2018). Holmes et al. (2016) and Delorme and Thomas (2019) have recently proposed that inertia gravity waves near the equator generate significant abyssal diapycnal mixing and upwelling. They might also preferentially release their momentum below the base of the mixed layer. It would be interesting to investigate whether this plays a significant role in the momentum balance along the equator and the turbulence below the mixed layer measured by Pujiana et al. (2018). If it does, the representation of these processes within ocean models would be an important issue.

Acknowledgments. Author Bell was supported by the Met Office Hadley Centre Climate Programme funded by BEIS and Defra. Authors Blaker and Hirschi acknowledge support from the NERC RAPID-AMOC project DYNAMOC (NE/M005097/1), the NERC project MESO-CLIP(NE/K005928/1), and the NERC project CLASS (NE/R015953/1). The authors gratefully acknowledge constructive suggestions from two anonymous reviewers and Andy White.

Data availability statement. The code and input files required to enable the reader to reproduce the results we present and to explore the oscillations further are available online (https:// doi.org/10.5281/zenodo.4415933).

\section{APPENDIX A}

\section{Calculation of the Boundary Terms}

The derivations presented in section 2 can be extended to cover the case when $\mathcal{A}$ and $\mathcal{B}$ are nonzero. Separating variables by extending (11) with

$$
\mathcal{A}=\sum_{m} \hat{p}_{m}(z) \tilde{\mathcal{A}}_{m} \quad \text { and } \quad \mathcal{B}=\sum_{m} \frac{d \hat{p}_{m}}{d z} \tilde{\mathcal{B}}_{m},
$$




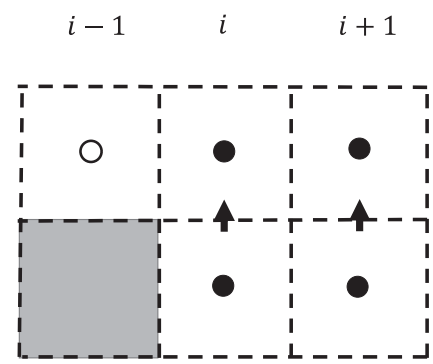

FIG. A1. Illustration of the differing points included in two zonal integrals. The zonal integral of $\partial / \partial t$ of (2) at the $v$ velocity points on model row $j+1 / 2$ includes the points marked by upward-pointing arrows. The zonal integral of $\partial^{2} h / \partial y \partial t$ at these points involves the values of $\partial h / \partial t$ at the tracer points marked by solid circles. The zonal integral of $\partial h / \partial t$ on row $j+1$ also includes the point at $i-1$, $j+1$ marked by an open circle. The shaded box is covered by land.

one can derive an extended form of (33) that includes $\mathcal{A}$ and $\mathcal{B}$ only in the form of their first-order time derivatives $\partial \mathcal{A} / \partial t$ and $\partial \mathcal{B} / \partial t$.

Diagnosing the additional forcing terms derived from $\mathcal{A}$ and $\mathcal{B}$ using the model outputs, however, is not entirely straightforward. This is partly because the model side boundaries follow the cell boundaries and hence have corners where their derivatives are discontinuous. So we describe here an approach that follows the calculations made by the numerical model more closely. This approach suggests that the terms corresponding to $\mathcal{A}$ and $\mathcal{B}$ are likely to provide mainly noisy forcing similar to that from the meridional winds (shown in Fig. 8). It could be used to calculate the additional forcing terms explicitly.

For brevity we consider only the terms (corresponding to $\mathcal{A}$ ) that arise from the zonal averaging of the meridional component of the momentum equations. We start our derivation from the zonally integrated shallow water equations [(16) and (17)] with $r$ set equal to zero:

$$
\begin{aligned}
\frac{\partial\left\langle u^{\prime}\right\rangle}{\partial t}-f\left\langle v^{\prime}\right\rangle & =\langle X\rangle+g\left(h_{W}^{\prime}-h_{E}^{\prime}\right), \\
\frac{\partial\left\langle v^{\prime}\right\rangle}{\partial t}+f\left\langle u^{\prime}\right\rangle & =\langle Y\rangle-g\left\langle\frac{\partial h^{\prime}}{\partial y}\right\rangle, \quad \text { and } \\
\frac{\partial\left\langle h^{\prime}\right\rangle}{\partial t}+H \frac{\partial\left\langle v^{\prime}\right\rangle}{\partial y} & =0 .
\end{aligned}
$$

Note that in (A3), the zonal integral applies to $\partial h^{\prime} / \partial y$ rather than to $h^{\prime}$. The numerical model's equations are formulated using the C-grid shown in Fig. A1 with (A3) calculated at the $v$ points marked by upward pointing arrows and (A4) calculated at the $h$ points (where tracers are held) marked by circles. The equations are solved by taking $\partial / \partial t$ of (A3) and substituting (A2) and (A4) into it. Terms corresponding to $\mathcal{A}$ in the continuous equations arise because at steps in the land-sea boundary, such as that at the land boundary between rows $j$ and $j+1$, the zonal integral of $\partial h^{\prime} / \partial y$ in (A3) does not contain the same grid points as the zonal integrals of $h^{\prime}$ at adjacent latitudes. In (A3), $\left\langle\partial h^{\prime} / \partial y\right\rangle$ at the $v$ points on row $j+1 / 2$ in Fig. A1 involves $h^{\prime}$ only at the points on rows $j$ and $j+1$ that are marked by solid circles. In (A4), $\left\langle h^{\prime}\right\rangle$ on row $j+1$ involves $h^{\prime}$ at the point marked by an open circle as well as the points marked by solid circles. The fact that \langle\rangle and $\partial / \partial y$ do not commute in this case gives rise to a correction to the zonal integral of $\partial h^{\prime} / \partial t$. The correction consists of the sum of $\partial h^{\prime} / \partial t$ terms at the points that lie adjacent to a boundary either to the north or the south of the row (the ones that would be marked by open circles following the notation of Fig. A1). So $\partial / \partial t$ of (A3), with (A2) and (A4) substituted into it, results in

$$
\begin{aligned}
\frac{\partial^{2}\left\langle\boldsymbol{v}^{\prime}\right\rangle}{\partial t^{2}}+f^{2}\left\langle\boldsymbol{v}^{\prime}\right\rangle-g H \frac{\partial^{2}\left\langle\boldsymbol{v}^{\prime}\right\rangle}{\partial y^{2}}= & f g\left(h_{E}^{\prime}-h_{W}^{\prime}\right)+g \sum_{x_{E_{i}}} \frac{\partial h_{E_{i}}^{\prime}}{\partial t} \frac{\Delta x_{E_{i}}}{\Delta y} \\
& -g \sum_{x_{W_{i}}} \frac{\partial h_{W_{i}}^{\prime}}{\partial t} \frac{\Delta x_{W_{i}}}{\Delta y}-f\langle X\rangle+\frac{\partial\langle Y\rangle}{\partial t} .
\end{aligned}
$$

Figure 7a shows that the improvement to the quality of the natural solutions $S_{T}$ when the first of the boundary pressure terms, $f g\left(h_{W}^{\prime}-h_{E}^{\prime}\right)$, is taken into account is large for some modes, particularly those with $(m, n)=(1,1)$ and $(2,1)$. This can be seen by comparing the black dots (natural solutions) for solutions of types $B$ and $D$. By contrast, the improvement between types $B$ and $D$ in the "half fit" solutions (red crosses), which allow adjustment of a constant offset, is very modest. This shows that the main impact of this pressure force on the solutions is due to a slowly varying zonal pressure difference, the slow mode solution, about which the solution oscillates. The additional boundary pressure forces probably have a relatively minor impact on the quality of the solutions because they involve only the time derivative of the pressures at the boundaries. The situation is likely to be similar to that in Fig. 8, which shows that the forcing by the zonal winds (red dots) and the boundary pressures provide coherent forcing whereas the forcing by the time derivative of the meridional winds (gray curve) fluctuates around zero in a noisy manner.

\section{APPENDIX B}

\section{A Least Squares Method to Optimize the Initial Conditions}

Suppose that we wish to choose the initial conditions to obtain the best fit solution to (37) for given values of $r, c_{m}$ and forcing. Because (37) is linear in $\tilde{\boldsymbol{v}}_{m, n}$, the sum of particular and homogeneous solutions of (37) are also solutions. We will find the linear combination that minimizes the square of the errors. To simplify notation, we drop the $m, n$ subscripts in what follows.

The particular solution $\tilde{v}_{p}$ of (37) that we use is the one with $\tilde{v}=\tilde{q}=0$ at the initial time. The homogeneous equation for (37) is

$$
\frac{d \tilde{v}}{d t}=\tilde{q}, \quad \frac{d \tilde{q}}{d t}=-2 r \tilde{q}-c^{2} \lambda \tilde{v}
$$

The first homogeneous solution $\tilde{v}_{A}$ that we use has $\tilde{v}_{A}=1$ and $\tilde{q}_{A}=0$. The second homogeneous solution $\tilde{v}_{B}$ has $\tilde{v}_{B}=0$ and $\tilde{q}_{B}=1$. Because (37) is linear in $\tilde{v}$, 


$$
\tilde{v}=\tilde{v}_{p}+A \tilde{v}_{A}+B \tilde{v}_{B}
$$

is also a solution of (37) for any choice of $A$ and $B$. So we use a least squares solver to minimize

$$
J_{2}=\sum_{k=1}^{K}\left(\tilde{v}_{k}-\tilde{v}_{p k}-\tilde{v}_{A k} A-\tilde{v}_{B k} B\right)^{2},
$$

The least squares solver provides the amplitudes of $A$ and $B$ that minimize $J_{2}$.

\section{REFERENCES}

Anderson, D. L. T., and P. B. Rowlands, 1976: The role of inertiagravity waves and planetary waves in the response of a tropical ocean to the incidence of an equatorial Kelvin wave on a meridional boundary. J. Mar. Res., 34, 295-312.

Andrews, T., and M. J. Webb, 2018: The dependence of global cloud and lapse rate feedbacks on the spatial structure of tropical Pacific warming. J. Climate, 31, 641-654, https:// doi.org/10.1175/JCLI-D-17-0087.1.

Balmaseda, M. A., K. Mogensen, and A. T. Weaver, 2013: Evaluation of the ECMWF ocean reanalysis system ORAS4. Quart. J. Roy. Meteor. Soc., 139, 1132-1161, https://doi.org/ 10.1002/qj.2063.

Bell, M. J., M. J. Martin, and N. K. Nichols, 2004: Assimilation of data into an ocean model with systematic errors near the equator. Quart. J. Roy. Meteor. Soc., 130, 873-893, https:// doi.org/10.1256/qj.02.109.

Blaker, A. T., J. J.-M. Hirschi, B. Sinha, B. A. de Cuevas, S. G. Alderson, A. C. Coward, and G. Madec, 2012: Large nearinertial oscillations of the Atlantic meridional overturning circulation. Ocean Modell., 42, 50-56, https://doi.org/10.1016/ j.ocemod.2011.11.008.

_, _ M. J. Bell, and A. Bokota, 2021: Wind-driven oscillations in meridional overturning circulations near the equator. Part I: Numerical simulations. J. Phys. Oceanogr., 51, 645-661, https://doi.org/10.1175/JPO-D-19-0296.1.

Blandford, R., 1966: Mixed gravity-Rossby waves in the ocean. Deep-Sea Res., 13, 941-961, https://doi.org/10.1016/00117471(76)90912-8.

Cane, M. A., and E. S. Sarachik, 1976: Forced baroclinic ocean motions: I. The linear equatorial unbounded case. J. Mar. Res., 34, 629-665.

— linear equatorial basin case. J. Mar. Res., 37, 355-398.

—, A. C. Clement, A. Kaplan, Y. Kushnir, D. Pozdnyakov, R. Seager, S. E. Zebiak, and R. Murtugudde, 1997: Twentiethcentury sea surface temperature trends. Science, 275, 957-960, https://doi.org/10.1126/science.275.5302.957.

Chapman, S., and R. S. Lindzen, 1970: Atmospheric Tides: Thermal and Gravitational. D. Reidel, $200 \mathrm{pp}$.

Clarke, A. J., 2008: An Introduction to the Dynamics of El Niño and the Southern Oscillation. Elsevier, $324 \mathrm{pp}$.

Clement, A. C., R. Seager, M. A. Cane, and S. E. Zebiak, 1996: An ocean dynamical thermostat. J. Climate, 9, 2190-2196, https:// doi.org/10.1175/1520-0442(1996)009<2190:AODT>2.0.CO;2.

Crawford, G. B., and W. G. Large, 1996: Numerical investigation of resonant inertial response of the ocean to wind forcing. J. Phys. Oceanogr., 26, 873-891, https://doi.org/10.1175/15200485(1996)026<0873:ANIORI >2.0.CO;2.

Cunningham, S. A., and Coauthors, 2007: Temporal variability of the Atlantic meridional overturning circulation at $26.5^{\circ}$ N. Science, 317, 935-938, https://doi.org/10.1126/science. 1141304.

Delorme, B. L., and L. N. Thomas, 2019: Abyssal mixing through critical reflection of equatorially trapped waves off smooth topography. J. Phys. Oceanogr., 49, 519-542, https://doi.org/ 10.1175/JPO-D-18-0197.1.

Farrar, J. T., and T. S. Durland, 2012: Wavenumber-frequency spectra of inertia-gravity and mixed Rossby-gravity waves in the equatorial Pacific Ocean. J. Phys. Oceanogr., 42, 18591881, https://doi.org/10.1175/JPO-D-11-0235.1.

Gill, A. E., 1982: Atmosphere-Ocean Dynamics. Academic Press, $662 \mathrm{pp}$.

Greatbatch, R. J., and Coauthors, 2018: Evidence for the maintenance of slowly varying equatorial currents by intraseasonal variability. Geophys. Res. Lett., 45, 1923-1929, https://doi.org/ 10.1002/2017GL076662.

Gruber, N., and Coauthors, 2009: Oceanic sources, sinks, and transport of atmospheric $\mathrm{CO}_{2}$. Global Biogeochem. Cycles, 23, GB1005, https://doi.org/10.1029/2008GB003349.

Hirschi, J. J.-M., A. Blaker, B. Sinha, B. de Cuevas, S. G. Alderson, A. C. Coward, and G. Madec, 2013: Chaotic variability of the meridional overturning circulation on subannual to interannual timescales. Ocean Sci, 9, 805-823, https://doi.org/10.5194/os-9-805-2013.

— , and Coauthors, 2020: The Atlantic meridional overturning circulation in high resolution models. J. Geophys. Res. Oceans, 125, e2019JC015522, https://doi.org/10.1029/2019JC015522.

Holmes, R. M., J. N. Moum, and L. N. Thomas, 2016: Evidence for seafloor-intensified mixing by surface-generated equatorial waves. Geophys. Res. Lett., 43, 1202-1210, https://doi.org/ 10.1002/2015GL066472.

Jochum, M., B. P. Briegleb, G. Danabasoglu, W. G. Large, N. J. Norton, S. R. Jayne, M. H. Alford, and F. O. Bryan, 2013: The impact of oceanic near-inertial waves on climate. J. Climate, 26, 2833-2844, https://doi.org/10.1175/JCLI-D-12-00181.1.

Johnson, H. L., P. Cessi, D. P. Marshall, F. Schloesser, and M. A. Spall, 2019: Recent contributions of theory to our understanding of the Atlantic meridional overturning circulation. J. Geophys. Res. Oceans, 124, 5376-5399, https://doi.org/ 10.1029/2019JC015330.

Kreyszig, E., 1979: Advanced Engineering Mathematics. John Wiley and Sons, $939 \mathrm{pp}$.

LaCasce, J. H., 2017: The prevalence of oceanic surface modes. Geophys. Res. Lett., 44, 11 097-11105, https://doi.org/10.1002/ 2017GL075430.

Large, W. G., and S. G. Yeager, 2009: The global climatology of an interannually varying air-sea flux data set. Climate Dyn., 33, 341-364, https://doi.org/10.1007/s00382-008-0441-3.

Lee, T., and J. Marotzke, 1998: Seasonal cycles of meridional overturning and heat transport of the Indian Ocean. J. Phys. Oceanogr., 28, 923-943, https://doi.org/10.1175/1520-0485(1998) 028<0923:SCOMOA $>2.0 . \mathrm{CO} ; 2$.

Lighthill, M. J., 1969: Dynamic response of the Indian Ocean to the onset of the southwest monsoon. Philos. Trans. Roy. Soc. London, 265A, 45-93, https://doi.org/10.1098/rsta.1969.0040.

Lozier, M. S., and Coauthors, 2019: A sea change in our view of overturning in the subpolar North Atlantic. Science, 363, 516521, https://doi.org/10.1126/science.aau6592.

Madden, R. A., 1979: Observations of large-scale traveling Rossby waves. Rev. Geophys. Space Phys., 17, 1935-1949, https:// doi.org/10.1029/RG017i008p01935.

Marshall, J., and K. Speer, 2012: Closure of the meridional overturning circulation through Southern Ocean upwelling. Nat. Geosci., 5, 171-180, https://doi.org/10.1038/ngeo1391. 
McCreary, J. P., 1985: Modeling equatorial ocean circulation. Annu. Rev. Fluid Mech., 17, 359-409, https://doi.org/10.1146/ annurev.fl.17.010185.002043.

McPhaden, M. J., and D. Zhang, 2002: Slowdown of the meridional overturning circulation in the upper Pacific Ocean. Nature, 415, 603-608, https://doi.org/10.1038/415603a.

Moore, D. W., 1968: Planetary-gravity waves in an equatorial ocean. Ph.D. thesis, Harvard University, 207 pp.

Mulholland, D. P., K. Haines, and M. A. Balmaseda, 2016: Improving seasonal forecasting through tropical ocean bias corrections. Quart. J. Roy. Meteor. Soc., 142, 2797-2807, https://doi.org/10.1002/qj.2869.

Park, J.-Y., C. A. Stock, X. Yang, J. P. Dunne, A. Rosati, J. John, and S. Zhang, 2018: Modeling global ocean biogeochemistry with physical data assimilation: A pragmatic solution to the equatorial instability. J. Adv. Model. Earth Syst., 10, 891-906, https://doi.org/10.1002/2017MS001223.

Pavlidis, T., 1982: Algorithms for Graphics and Image Processing. Computer Science Press, $416 \mathrm{pp}$

Pujiana, K., J. N. Moum, and W. D. Smyth, 2018: The role of turbulence in redistributing upper-ocean heat, freshwater, and momentum in response to the $\mathrm{MJO}$ in the equatorial Indian Ocean. J. Phys. Oceanogr., 48, 197-220, https://doi.org/ 10.1175/JPO-D-17-0146.1.

Sloyan, B. M., and S. R. Rintoul, 2001: Circulation, renewal, and modification of Antarctic Mode and Intermediate Water. J. Phys. Oceanogr., 31, 1005-1030, https://doi.org/10.1175/ 1520-0485(2001)031<1005:CRAMOA>2.0.CO;2.
Smeed, D. A., and Coauthors, 2018: The North Atlantic Ocean is in a state of reduced overturning. Geophys. Res. Lett., 45, 1527-1533, https://doi.org/10.1002/2017GL076350.

Song, L., Y. Li, J. Wang, F. Wang, S. Hu, C. Liu, X. Diao, and C. Guan, 2018: Tropical meridional overturning circulation observed by subsurface moorings in the western Pacific. Sci. Rep., 8, 7632, https://doi.org/10.1038/s41598018-26047-7.

Thuburn, J., and T. J. Woollings, 2005: Vertical discretizations for compressible Euler equation atmospheric models giving optimal representation of normal modes. J. Comput. Phys., 203, 386-404, https://doi.org/10.1016/j.jcp.2004.08.018.

Vallis, G. K., 2017: Atmospheric and Oceanic Fluid Dynamics. Cambridge University Press, 946 pp., https://doi.org/10.1017/ 9781107588417.

Vecchi, G. A., and B. J. Soden, 2007: Global warming and the weakening of the tropical circulation. J. Climate, 20, 43164340, https://doi.org/10.1175/JCLI4258.1.

Waters, J., D. Lea, M. Martin, I. Mirouze, A. Weaver, and J. While, 2014: Implementing a variational data assimilation system in an operational 1/4 degree global ocean model. Quart. J. Roy. Meteor. Soc., 141, 333-349, https://doi.org/10.1002/ qj.2388.

Zhang, D., and M. J. McPhaden, 2006: Decadal variability of the shallow Pacific meridional overturning circulation: Relation to tropical sea surface temperatures in observations and climate change models. Ocean Modell., 15, 250-273, https://doi.org/ 10.1016/j.ocemod.2005.12.005. 